\title{
H I AND CO IN BLUE COMPACT DWARF GALAXIES: HARO 2 AND HARO 4
}

\author{
H. Bravo-Alfaro \\ Departamento de Astronomía, Universidad de Guanajuato, Apdo. Postal 144, Guanajuato 36000, Mexico \\ E. BRINKS \\ Instituto Nacional de Astrofísica, Optica y Electrónica, Apdo. Postal 51 and 216, Puebla 72000, Mexico \\ A. J. BAKER ${ }^{1}$ \\ Owens Valley Radio Observatory, MS 105-24, California Institute of Technology, Pasadena, CA 91125 \\ F. WALTER \\ National Radio Astronomy Observatory, P.O. Box O, Socorro, NM 87801 \\ AND \\ D. KunTH \\ Institut d'Astrophysique, 98bis Boulevard Arago, F-75014 Paris, France \\ Received 2003 May 21; accepted 2003 September 24
}

\begin{abstract}
We present Very Large Array H i imaging of two blue compact dwarf galaxies, Haro 2 and Haro 4, and of the spiral galaxy Haro 26, which is projected some 22' southwest of Haro 4. We also show a map of the CO(1-0) distribution of Haro 2 obtained with the Owens Valley Radio Observatory Millimeter Array, as well as derive an upper limit for $\mathrm{CO}(2-1)$ emission from Haro 4 obtained with the Caltech Submillimeter Observatory. The H I data of Haro 2 reveal that the kinematical major axis lies perpendicular to the photometric major axis, indicating that the atomic hydrogen rotates about the major axis of the galaxy. This confirms earlier indications based on CCD photometry that Haro 2 is a dust-lane dE rather than a dIrr. We propose that the present neutral and molecular ISM configuration is due to recent gas accretion or a merger. The $\mathrm{H}$ I distribution and dynamics of Haro 4 and the neighboring spiral Haro 26 suggest that they are currently undergoing a tidal interaction, reinforcing the notion that interactions play an important role in triggering the star formation witnessed in blue compact galaxies.
\end{abstract}

Key words: galaxies: dwarf — galaxies: individual (Haro 2, Haro 4, Haro 26) — radio lines: galaxies

\section{INTRODUCTION}

Blue compact dwarf (BCD) galaxies are dwarf irregular systems that are currently experiencing a burst of star formation. Their appearance is dominated by the starburst, which appears compact and blue, hence their name. Their spectra show all the characteristics of $\mathrm{H}$ II regions, which has led many BCDs to be classified as H II galaxies (although, in general, not all $\mathrm{H}$ II galaxies are necessary dwarf systems). At their current star formation rates (SFRs), these systems would exhaust their total available gas in considerably less than a Hubble time; for this reason, it is generally accepted that their star formation must be episodic.

Gas-rich dwarf galaxies play an important role in our attempts to understand galaxy formation and evolution. BCDs show low metallicities, which suggests that they are chemically unevolved systems. They are more metal-deficient than the solar neighborhood by factors between 3 and 20 (Kunth \& Östlin 2000), with a few objects such as I Zw 18 (Searle \& Sargent 1972) and SBS 0335-052 (Melnick, Heydari-Malayeri, \& Leisy 1992) reaching metallicities as low as $0.025 Z_{\odot}$.

This said, most BCDs do not seem to be young objects in the sense that they are undergoing their first episode of star formation. Many reveal older stellar populations underlying the bright young stars produced in the current bursts

\footnotetext{
${ }^{1}$ Current address: Max-Planck-Institut für extraterrestrische Physik, Postfach 1312, D-85741 Garching, Germany.
}

(Papaderos et al. 1996; Östlin 2000; Tosi 2001). This poses some interesting questions regarding the production and possible loss of heavy elements. Recent X-ray observations with the Chandra X-Ray Observatory seem to indicate that in starbursting dwarf galaxies, such as NGC 1569 and NGC 3077 (Martin, Kobulnicky, \& Heckman 2002; Ott, Martin, \& Walter 2003), blowout of material into the halo occurs that will remove the products of nucleosynthesis in stars more massive than $8 M_{\odot}$ and disperse those across a larger volume. Numerical simulations have suggested that in extreme cases blow-away can occur, which removes these products from the galaxy proper and enriches the intergalactic medium (Mac Low \& Ferrara 1999).

It is not clear what causes a BCD to initiate a burst or episode of star formation, and several mechanisms have been proposed, ranging from internal instabilities to external (especially tidal) triggers. Reports that some BCDs are isolated systems (e.g., van Zee, Skillman, \& Salzer 1998, who observed four such objects in H I) suggests that interactions may not be the only explanation. But other studies (e.g., Taylor et al. 1994; Taylor 1997; Walter et al. 1997; Pustilnik et al. 2001b, and references therein) have shown that the presence of close companions producing tidal interactions may be a sufficient condition to explain BCD bursts. Furthermore, recent studies (see, e.g., Noeske et al. 2001) of large samples of star-forming dwarf galaxies that look for faint companions give additional support to the hypothesis of interaction-induced star formation in BCDs. 
TABLE 1

Optical Properties of the Galaxies Observed in H i

\begin{tabular}{|c|c|c|c|c|c|c|c|c|}
\hline ID & Alias & $\alpha_{\mathrm{J} 2000.0}$ & $\delta_{\mathrm{J} 2000.0}$ & Morph. Type & $M_{B}$ & $\begin{array}{c}D \\
(\mathrm{Mpc})\end{array}$ & $12+\log [\mathrm{O} / \mathrm{H}]$ & Ref. \\
\hline Haro $2 \ldots \ldots$. & Mrk 33 & 103231.9 & +542403 & H II Im pec & -18.1 & $19.5^{\mathrm{a}}$ & 8.4 & 1 \\
\hline Haro 4 ........ & Mrk 36 & 110458.5 & +290822 & $\mathrm{BCD}$ & -14.7 & $6.9^{\mathrm{b}}$ & 7.8 & 2 \\
\hline Haro $26 \ldots . .$. & NGC 3510 & 110343.6 & +285306 & $\mathrm{SB}(\mathrm{s}) \mathrm{m}$ & -17.2 & $7.9^{\mathrm{b}}$ & $\ldots$ & $\ldots$ \\
\hline
\end{tabular}

NoтE.-Units of right ascension are hours, minutes, and seconds, and units of declination are degrees, arcminutes, and arcseconds.

${ }^{a}$ Assuming $H_{0}=75 \mathrm{~km} \mathrm{~s}^{-1} \mathrm{Mpc}^{-1}$.

${ }^{\mathrm{b}}$ From Tully 1988.

References.-(1) Mas-Hesse \& Kunth 1999; (2) Izotov \& Thuan 1999.

Most studies to date have focused, broadly speaking, on the optical characteristics of BCDs, with several incorporating $\mathrm{H}_{\mathrm{I}}$ observations of sufficiently high spatial resolution to resolve the objects under study. Despite the fact that gas has to pass through a molecular phase for star formation to commence, only very few authors have reported high-resolution (interferometric) maps of molecular gas in BCDs. Detecting CO in dwarf galaxies has proved to be quite a challenge, a fact that is frequently attributed to the low metallicity in these objects (Gondhalekar et al. 1998; Taylor, Kobulnicky, \& Skillman 1998; Barone et al. 2000; Meier et al. 2001b). As a result, only a few dwarfs have been clearly detected in $\mathrm{CO}$, and still fewer objects have been mapped with millimeter interferometers (NGC 5253: Turner, Beck, \& Hurt 1997; Meier, Turner, \& Beck 2002; NGC 1569: Taylor et al. 1999; NGC 4214: Walter et al. 2001; NGC 3077: Meier, Turner, \& Beck 2001a; Walter et al. 2002; IC 10: Walter et al. 2004). With the advent of the FUSE telescope, $\mathrm{H}_{2}$ molecules have been searched for in BCDs, but upper limits for the diffuse $\mathrm{H}_{2}$ column density are found to be very low, with $N\left(\mathrm{H}_{2}\right) \lesssim 10^{15} \mathrm{~cm}^{-2}$ (Vidal-Madjar et al. 2000).

To increase the sample of galaxies studied in reasonable detail, we have mapped the BCDs Haro 2 (=Mrk 33=UGC 5720) and Haro 4 (=Mrk 36) in $\mathrm{H}$ I. In addition, we have obtained an interferometric map of Haro 2 in the $\mathrm{CO}(1-0)$ line. Haro 2 is cataloged as a Wolf-Rayet galaxy (Beck, Turner, \& Kovo 2000; Kunth \& Joubert 1985) and has been extensively studied as a key object for understanding star formation activity in low-metallicity environments. It is also one of the few BCDs that has been detected in $\mathrm{CO}(1-0)$, $\mathrm{CO}(2-1)$, and $\mathrm{CO}(3-2)$ single-dish observations (Sage et al. 1992; Israel, Tacconi, \& Baas 1995; Barone et al. 2000; Meier et al. 2001b).

This paper is organized in the usual way. In $\S 2$ we describe the $\mathrm{H} \mathrm{I}$ and $\mathrm{CO}$ observations. The $\mathrm{H}$ I distributions and kinematics of the galaxies are shown in $\S 3$, where we also present the results derived from the CO observations. In $\S 4$ we discuss the atomic and molecular gas contents and distributions of Haro 2 and Haro 4, and the implications for BCD evolution. A summary of our results is presented in $\S 5$.

\section{OBSERVATIONS}

Table 1 summarizes the optical properties of the two galaxies under study plus some relevant data on Haro 26 (=NGC 3510), which lies at a separation of $\sim 22^{\prime}$ southwest of Haro 4 . We mapped the $\mathrm{H}$ I in Haro 2 and the Haro 4/Haro 26 system with the $\mathrm{NRAO}^{2}$ Very Large Array (VLA). Haro 2 was mapped in the $\mathrm{CO}(1-0)$ rotational transition with the Owens Valley Radio Observatory (OVRO) millimeter array. Single-dish CO(2-1) observations of Haro 4 were obtained at the Caltech Submillimeter Observatory (CSO) on Mauna Kea, Hawaii. In this section we describe the procedures used to obtain and reduce the data.

\subsection{H I Imaging}

The H I data were obtained with the VLA in its C and D configurations, during two runs carried out in 1994 November and in 1995 May, respectively (see Table 2 for the VLA observing $\log$ ). Two pointings were used for each run, one centered on Haro 2 and the other on Haro 4, but including within the primary beam the galaxy Haro 26 . We observed with the correlator in mode 4, resulting in two data sets-one with 63 channels at $5.2 \mathrm{~km} \mathrm{~s}^{-1}$ resolution and the other with 31 channels at $20.8 \mathrm{~km} \mathrm{~s}^{-1}$ resolution. Hanning smoothing was applied on-line during the observations. After inspecting both data sets, we decided to work only with the lower velocity resolution data because of insufficient signal-to-noise ratio at high velocity resolution. Both $\mathrm{C}$ - and $\mathrm{D}$-configuration data were combined, leading to a spatial resolution of about $14^{\prime \prime} \times 15^{\prime \prime}$.

\footnotetext{
2 The National Radio Astronomy Observatory is a facility of the National Science Foundation operated under cooperative agreement by Associated Universities, Inc.
}

TABLE 2

VLA H i OBSERVING LoG

\begin{tabular}{cccccr}
\hline \hline Field & $\alpha_{\mathrm{J} 2000.0}$ & $\delta_{\mathrm{J} 2000.0}$ & Date & VLA Config. & $\begin{array}{c}t_{\text {int }} \\
\text { (minutes) }\end{array}$ \\
\hline Haro 2 ........ & 103230 & +542500 & 1994 Nov 1 & $\mathrm{C}$ & 193 \\
Haro 2 ....... & 103230 & +542500 & 1995 May 2 & $\mathrm{D}$ & 22 \\
Haro 4/26.... & 110500 & +290900 & 1994 Nov 1 & $\mathrm{C}$ & 202 \\
Haro 4/26.... & 110500 & +290900 & 1995 May 2 & $\mathrm{D}$ & 32 \\
\hline
\end{tabular}


TABLE 3

Parameters of the H i Maps Made from the Combined C+D Configuration Data

\begin{tabular}{|c|c|c|c|c|c|}
\hline $\begin{array}{l}\text { Field } \\
\text { (1) }\end{array}$ & $\begin{array}{l}\text { Beam Size } \\
\left(\operatorname{arcsec}^{2}\right) \\
(2)\end{array}$ & $\begin{array}{l}\text { Beam Size } \\
\quad(\mathrm{kpc}) \\
(3)\end{array}$ & $\begin{array}{c}\Delta \nu_{\text {chan }} \\
(\mathrm{kHz}) \\
(4)\end{array}$ & $\begin{array}{c}\mathrm{rms} \\
\left(\mathrm{mJy} \text { beam }^{-1}\right) \\
(5)\end{array}$ & $\begin{array}{l}\mathrm{rms} \\
\left(T_{b}\right) \\
(\mathrm{K}) \\
(6)\end{array}$ \\
\hline Haro $2 \ldots \ldots \ldots$. & $14.8 \times 14.1$ & 1.4 & 96.6 & 0.37 & 1.1 \\
\hline Haro $4 / 26 \ldots .$. & $15.5 \times 14.0$ & 0.5 & 96.6 & $0.39 / 1.95$ & $1.1 / 5.4$ \\
\hline
\end{tabular}

Standard VLA calibration and imaging procedures were applied, using the NRAO astronomical image processing system (AIPS; see van Moorsel, Kemball, \& Greisen 1996) for an up-to-date description). Data cubes were produced with robust weighting (Briggs 1995) in order to maximize sensitivity while preserving as high a spatial resolution as possible. In order to define the extent of the $\mathrm{H}$ i emission, we initially averaged four line-free channels on either side of the band to create a first approximation of the continuum. The corresponding image was subtracted from the channel maps, resulting in a cube of $\mathrm{H}$ I line emission only. We used this cube to conduct a more thorough search for $21 \mathrm{~cm}$ line emission. Following this search, a new continuum image was made using all channels deemed free from line emission. After continuum subtraction the channels containing line emission were CLEANed. To build moment maps from the data cubes, we used a masking technique: for each CLEANed cube with a beam of around $15^{\prime \prime}$ we applied a convolution to a beam approximately twice as large, i.e., $30^{\prime \prime}$. The smoothed cubes were then clipped at a level of $2 \sigma$, and $\mathrm{H}$ I emission was identified via visual inspection on the basis of spatial continuity in neighboring channel maps. A conditional transfer (or mask) was applied to the original CLEANed cube using the blanked, smoothed data. As a last step H I surface brightness maps and velocity fields were created.

The final cubes for Haro 2, targeted at the center of the observed field, have an rms per channel of $0.37 \mathrm{mJy}^{\text {beam }}{ }^{-1}$. The cubes for Haro 4 and Haro 26 have an rms per channel of $0.39 \mathrm{mJy}$ beam $^{-1}$ at the pointing center, which lies close to the position of Haro 4. At the position of Haro 26, the correction for primary-beam attenuation elevates the rms by a factor of 5 , approximately. The observations have on average an $\mathrm{H}$ I mass detection threshold for individual clouds of $4.5 \times 10^{6} M_{\odot}$ and $1.1 \times 10^{6} M_{\odot}$ for Haro 2 and Haro 4, respectively. These values correspond to surface brightness sensitivities of $2.5 \times 10^{20} \mathrm{~cm}^{-2}$, where we have assumed that a signal is real if it shows up at the $3 \sigma$ level over at least two consecutive channels. As a by-product of the $\mathrm{H}$ I observations, we obtain useful information about the radio continuum emission coming from the three galaxies as well (see below). The $\mathrm{H}$ I observational parameters of the combined $\mathrm{C}$ - and $\mathrm{D}$-configuration data are listed in Table 3, where columns (2) and (3) give the beam size in seconds of arc and the linear resolution in kiloparsecs, respectively. Column (4) gives the channel width, column (5) the rms noise per channel after continuum subtraction, and column (6) the noise expressed in units of Kelvin brightness temperature.

\subsection{CO Observations}

We observed the $\mathrm{CO}(1-0)$ transition in Haro 2 with the $\mathrm{L}$ and $\mathrm{H}$ configurations of the OVRO millimeter array (Padin et al. 1991) during the 1995-1996 season. Table 4 summarizes our observations. The array includes six $10.4 \mathrm{~m}$ diameter antennas with half-power beamwidths of $60^{\prime \prime}$ at $115 \mathrm{GHz}$. Two separate correlators processed continuum and spectral line data: an analog correlator recorded continuum bandwidths of $1 \mathrm{GHz}$ centered at $\pm 1.5 \mathrm{GHz}$ relative to the reference local oscillator frequency, while a digital correlator provided 112 frequency channels in the upper sideband, Hanning-smoothed to $4 \mathrm{MHz}$ $\left(10.4 \mathrm{~km} \mathrm{~s}^{-1}\right)$ resolution.

We calibrated the data within the OVRO millimeter array database using the MMA package (Scoville et al. 1993). Observations of Uranus obtained with similar elevation and atmospheric decorrelation were used to determine the flux density of the bright quasar $1150+497$ at each epoch; we estimate that the resulting uncertainty in our flux scale is $\sim 10 \%$. Paired observations of $1150+497$ interleaved with observations of Haro 2 every 30-40 minutes were then used to remove the effects of instrumental and atmospheric variations on phase and amplitude gains. Observations of bright quasars were used for passband calibration.

After editing the visibilities in the DIFMAP package (Shepherd 1997), our final data set included $24.3 \mathrm{hr}$ of integration and 30 distinct baselines. We mapped the data using the IMAGR task in AIPS, cleaning a single large region down to the rms noise in each velocity channel. Maps of the $1 \mathrm{GHz}$ continuum channel in the line-free sideband showed no evidence of emission (with a $3 \sigma$ limit of $2.3 \mathrm{mJy}$ at $112 \mathrm{GHz}$ ), so subtraction of continuum from the spectral line data was unnecessary. Natural weighting produced a synthesized beam of 3 ." $3 \times 2$." 6 at position angle $-84^{\circ} .0$ and an rms noise per channel of $9.6 \mathrm{mJy}$ beam $^{-1}$.

We observed Haro 4 in the $\mathrm{CO}(2-1)$ transition at the CSO on 2001 April 8. The beam size of the $\mathrm{CSO}$ at $230 \mathrm{GHz}$ is

TABLE 4

OVRO CO(1-0) ObServing Log for Haro 2

\begin{tabular}{ccccc}
\hline \hline Date & Array Config. & $\begin{array}{c}\text { Longest Baseline } \\
(\mathrm{m})\end{array}$ & Passband Calibrator & $\begin{array}{c}\text { 1150+497 Strength } \\
(\text { Jy) }\end{array}$ \\
\hline 1995 Oct $5 \ldots \ldots$. & L & 115 & $3 \mathrm{C} 84$ & 1.0 \\
1995 Dec $17 \ldots .$. & $\mathrm{H}$ & 242 & $0528+134$ & 0.8 \\
1996 May $11 \ldots . .$. & $\mathrm{L}$ & 115 & $3 \mathrm{C} 273$ & 0.9 \\
\hline
\end{tabular}




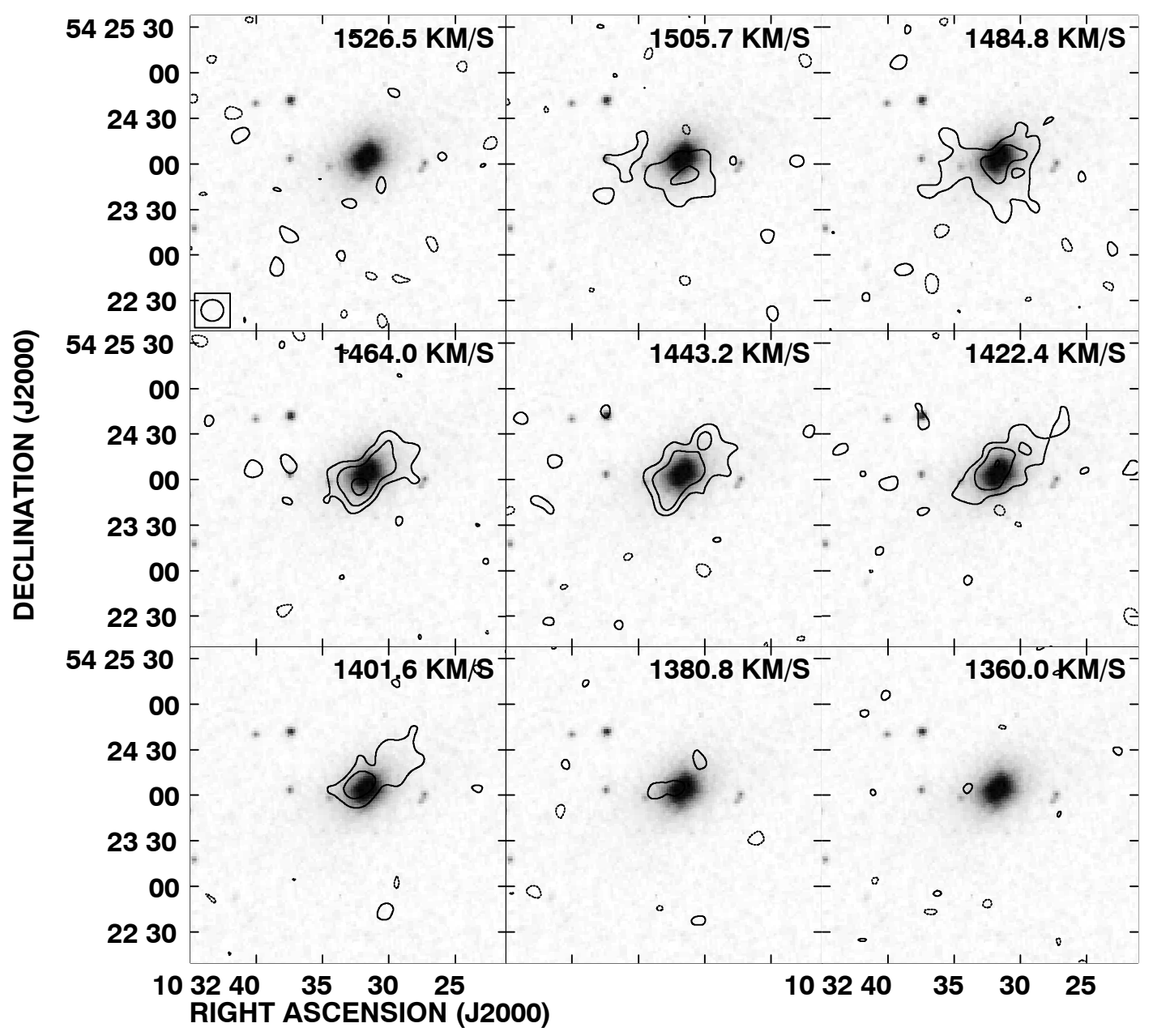

Fig. 1.-Mosaic of the channels containing line emission from Haro 2, each superposed on a DSS $B$-band gray-scale image. Contours are $-2.5,2.5,5.0$, and 10 $\sigma$; negative contours are dashed. The beam size indicated in the top left panel is $14{ }^{\prime \prime} 8 \times 14^{\prime \prime} .1$.

$\sim 30^{\prime \prime}$, and the main beam efficiency is determined from observations of Mars to be $\eta_{\mathrm{mb}}=0.72$ (Gerin \& Phillips 2000). The 1024 channel $500 \mathrm{MHz}$ AOS spectrometer was used for the observations. The system temperature was $630 \mathrm{~K}$ $\left(\tau_{225 \mathrm{GHz}}=0.2\right)$. In total, $1800 \mathrm{~s}$ were spent on-source, leading to an rms of $T_{A}^{*}=9 \mathrm{mK}\left(T_{\mathrm{mb}}=12.5 \mathrm{mK}\right)$ in a $6 \mathrm{~km} \mathrm{~s}^{-1}$ Hanning-smoothed channel. No signal was detected in our observations.

\section{RESULTS}

\subsection{H I Morphology and Kinematics of Haro 2}

This is the first extensive interferometric $\mathrm{H}_{\mathrm{I}}$ work on Haro 2; previous $\mathrm{H}$ I results were restricted to single-dish studies. Like most star-forming dwarf galaxies, Haro 2 is $\mathrm{H} \mathrm{I}$-rich. Figure 1 presents the channel maps. These show clearly resolved structures that are individually oriented in a southeast-northwest direction and that show a slight displacement perpendicular to this direction as a function of velocity.

The H i parameters we obtain are listed in Table 5. Column (1) gives the galaxy name; columns (2) and (3) list the velocity centroid and FWHM. We conservatively take half the channel separation $\left(10.3 \mathrm{~km} \mathrm{~s}^{-1}\right)$ as the uncertainty for the $\mathrm{H}$ I central (systemic) velocity. The error in the velocity width is estimated to be of order a quarter of a channel, or $\approx 5 \mathrm{~km} \mathrm{~s}^{-1}$. The integrated $\mathrm{H}$ I flux (corrected for primary-beam attenuation) is listed in column (4); we estimate an error of about $10 \%$ for the absolute flux calibration. The radio continuum flux determined

TABLE 5

Measured H i and 20 Centimeter Radio Continuum Parameters

\begin{tabular}{|c|c|c|c|c|c|c|}
\hline $\begin{array}{l}\text { ID } \\
\text { (1) }\end{array}$ & $\begin{array}{c}v_{c} \\
\left(\mathrm{~km} \mathrm{~s}^{-1}\right) \\
(2)\end{array}$ & $\begin{array}{c}\Delta v_{50} \\
\left(\mathrm{~km} \mathrm{~s}^{-1}\right) \\
(3)\end{array}$ & $\begin{array}{c}F_{\mathrm{H}} \\
\left(\mathrm{Jy} \mathrm{km} \mathrm{s}^{-1}\right) \\
(4)\end{array}$ & $\begin{array}{c}S_{1.4 \mathrm{GHz}} \\
(\mathrm{mJy}) \\
(5)\end{array}$ & $\begin{array}{c}M_{\mathrm{H}_{\mathrm{I}}} \\
\left(10^{8} \mathrm{M}_{\odot}\right) \\
(6)\end{array}$ & $\begin{array}{c}N^{\text {peakH I }} \\
\left(10^{21} \mathrm{~cm}^{-2}\right) \\
(7)\end{array}$ \\
\hline Haro $2 \ldots \ldots . . .$. & 1443 & 111 & 2.6 & 24.6 & 2.3 & 2.0 \\
\hline Haro $4 \ldots \ldots . . .$. & 650 & 43 & 1.1 & 3.4 & 0.2 & 2.4 \\
\hline Haro $26 \ldots . . . .$. & 712 & 187 & 40 & 23.8 & 8.5 & 2.2 \\
\hline
\end{tabular}


from the line-free channels is given in column (5), and the integrated H I mass in column (6). Column (7) gives the observed peak H I column density.

Comparison of our $\mathrm{H}$ I detection with published single-dish data yields the following results. Gordon \& Gottesman (1981) observed Haro 2 with the 300 foot $(30.5 \mathrm{~m})$ Green Bank Telescope, which had a 10.8 beam. From examining their published spectra, we deduce a peak flux of $34 \mathrm{mJy}$ and a line width at $50 \%$ of the peak of $\Delta v_{50} \approx 100 \mathrm{~km} \mathrm{~s}^{-1}$. The authors publish a line width at $20 \%$ of the peak of $\Delta v_{20}=208 \mathrm{~km} \mathrm{~s}^{-1}$ and a total $\mathrm{H}_{\text {I }}$ mass of $5.2 \times 10^{8} M_{\odot}$ (using a distance of $21 \mathrm{Mpc}$ ). If we adjust their distance to the one used throughout this paper $(19.5 \mathrm{Mpc})$, the total $\mathrm{H}$ I mass is $4.2 \times 10^{8} M_{\odot}$. In a more recent study with the Effelsberg $100 \mathrm{~m}$ telescope, which gives a 9.3 beam, Huchtmeier, Sage, \& Henkel (1995) report $\Delta v_{50}=110 \mathrm{~km} \mathrm{~s}^{-1}, \Delta v_{20}=170 \mathrm{~km} \mathrm{~s}^{-1}$, and a peak flux of $34.0 \pm 3.4 \mathrm{mJy}$. These authors point out that their observations of Haro 2 may suffer from confusion with a close companion located 4.'7 to the east (see also Nilson 1973). We inspected our data cube around the position of the putative companion but detected no $\mathrm{H}$ I emission. It is therefore justified to use their single-dish data to derive an $\mathrm{H}$ i mass for Haro 2, which (integrating the published spectra by hand) comes out to be $3.6 \times 10^{8} M_{\odot}$.

The two single-dish results agree with each other within their respective uncertainties. The single-dish line widths also agree with the value we determine from our interferometric data, but the latter fall short as far as the integrated flux is concerned: the VLA recovers an $\mathrm{H}$ I mass of order $2.3 \times 10^{8} M_{\odot}$, i.e., only $\sim 55 \%$ of the single-dish mass (see Table 5). We are aware of the fact that CLEANed maps do not always yield accurate integrated fluxes, especially when the $\mathrm{H}$ I distribution in the channel maps is extended on scales comparable to or larger than $\sim 50 \%$ of the primary beam (see, e.g., Jörsäter \& van Moorsel 1995). In the case of Haro 2, whose H I emission has an extent of order $1^{\prime}-2^{\prime}$, or less than $1 / 15$ th of the primary beam, we discard this as a possible explanation. Instead, we postulate that the VLA maps only reveal the "tip of the iceberg" and that the galaxy contains extended, low surface brightness gas that is mostly missed by the interferometer.

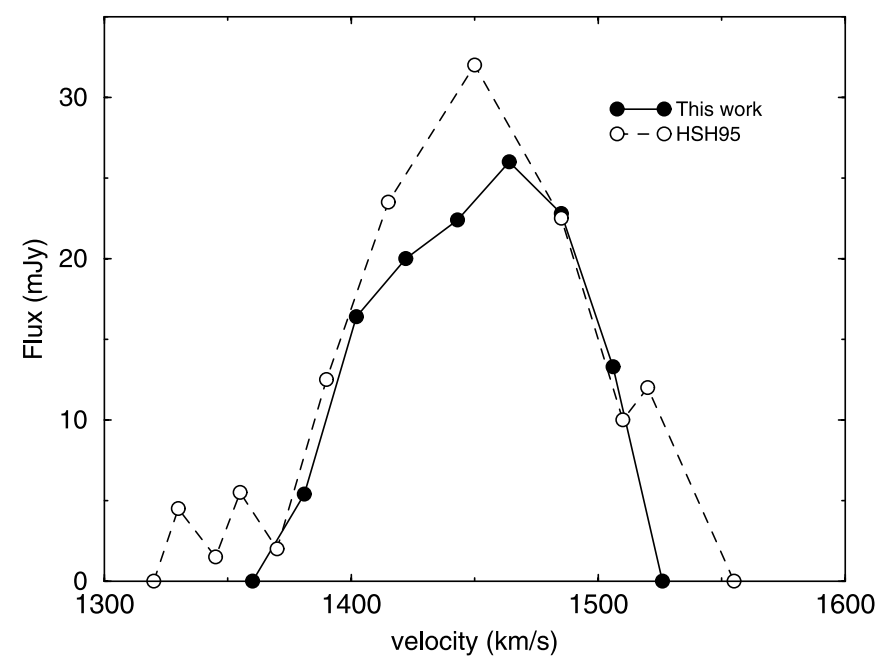

FIG. 2.-Global $\mathrm{H}$ I profile for Haro 2 obtained by integrating the channel maps over the area of the source after continuum subtraction, blanking, and correction for primary-beam attenuation. For a comparison with single-dish $\mathrm{H}$ I data, we overplot the profile (labeled "HSH95") obtained with the $100 \mathrm{~m}$ Effelsberg telescope by Huchtmeier et al. (1995).

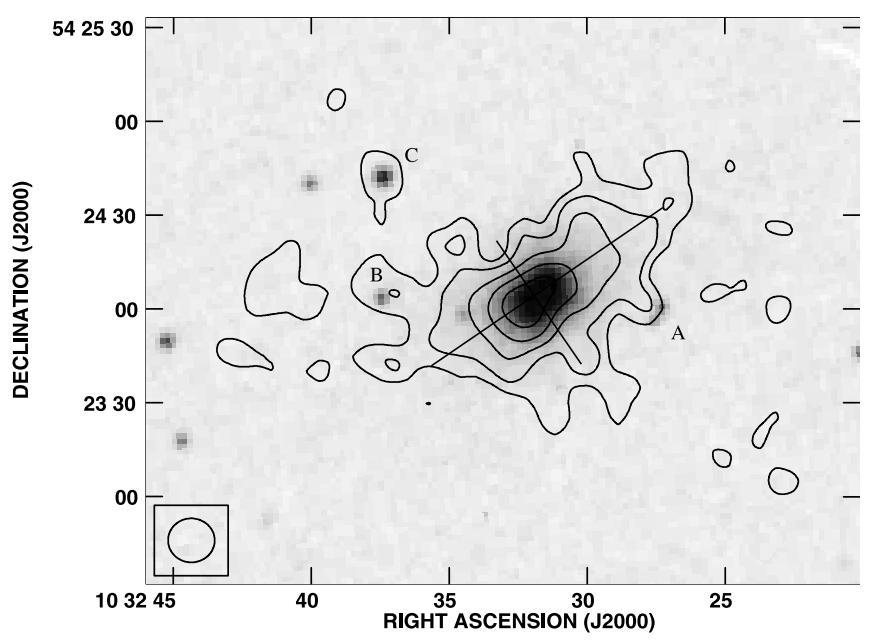

FIG. 3.- H I surface density contours for Haro 2, superposed on a DSS $B$-band gray-scale image. The contours are $2.0(5 \sigma), 4.0,8.1,12.2$, and $16.2 \times 10^{20} \mathrm{~cm}^{-2}$. The size of the synthesized beam is $14 . " 8 \times 14$." 1 . Three marginally detected $\mathrm{H}$ I clouds, which seem to coincide with optical counterparts, are identified (A, B, and C; see $\S 3.1$ ). Solid lines indicate the directions along which the $\mathrm{H}$ I major and minor axes were measured.

Figure 2 shows the integrated spectrum from our VLA data. This profile is in very good agreement with that obtained by Huchtmeier, Sage, \& Henkel (1995) in terms of both the velocity width and the slight asymmetry toward lower velocity side, where a bump appears near $1420 \mathrm{~km} \mathrm{~s}^{-1}$. We do not confirm the secondary peak seen by Gordon \& Gottesman (1981) at $1550 \mathrm{~km} \mathrm{~s}^{-1}$; this feature was also not detected by Huchtmeier et al. (1995) at Effelsberg. In Figure 2 it is also clear that we are missing part of the extended emission of the galaxy.

Figure 3 is a map at $15^{\prime \prime}$ resolution of the $\mathrm{H}_{\mathrm{I}}$ surface brightness in Haro 2 superposed on an optical DSS image in gray scale. It appears elongated in a southeast-northwest direction, much like what can be seen in the channel maps. The $\mathrm{H}$ I disk dimensions are 1.'4 $\times 0 .{ }^{\prime} 7$ (equivalent to $7.9 \mathrm{kpc} \times$ $4.0 \mathrm{kpc}$ at the distance of Haro 2) at a level of $\sim 5 \times 10^{20} \mathrm{~cm}^{-2}$. These are estimated along the optical major and minor axis of the galaxy, respectively, as shown by the two lines drawn in Figure 3, but they were measured from a smoothed $\mathrm{H}_{\text {I }}$ map that is not shown in this work. The $\mathrm{H}$ I disk orientation roughly coincides with the $1.0 \times 00^{\prime} 9(5.7 \mathrm{kpc} \times 5.1 \mathrm{kpc})$ optical disk, whose major axis has a position angle of approximately $135^{\circ}$. At least three features appear to be unresolved $\mathrm{H}$ i clouds with optical counterparts: one to the west of Haro 2 (labeled "A," at $\left.\alpha_{\mathrm{J} 2000.0}=10^{\mathrm{h}} 32^{\mathrm{m}} 27^{\mathrm{s}}, \delta_{\mathrm{J} 2000.0}=+54^{\circ} 24^{\prime} 00^{\prime \prime}\right)$, one to the east (labeled "B," at $\alpha_{\mathrm{J} 2000.0}=10^{\mathrm{h}} 32^{\mathrm{m}} 37^{\mathrm{s}}, \delta_{\mathrm{J} 2000.0}=+54^{\circ} 24^{\prime} 00^{\prime \prime}$ ), and one to the northeast (labeled " $\mathrm{C}$," at $\alpha_{\mathrm{J} 2000.0}=10^{\mathrm{h}} 32^{\mathrm{m}} 37^{\mathrm{s}}$, $\delta_{\mathrm{J} 2000.0}=+54^{\circ} 24^{\prime} 43^{\prime \prime}$ ). These H I concentrations may either be dynamically distinct structures or represent only the local peaks of a smooth extended $\mathrm{H}$ i component that the VLA otherwise resolves out. In either case, although each of the peaks is detected in at least two velocity channels, more sensitive observations will be needed to confirm their reality.

Figure 4 presents the $\mathrm{H}$ I velocity field of Haro 2 and reveals the galaxy's complex kinematics. There is virtually no velocity gradient visible along the major axis defined by the optical light and $\mathrm{H}$ I distribution; a cursory inspection of the channel maps (Fig. 1) already hints at this behavior. A clear gradient is seen at roughly right angles to the major axis, with only a weak suggestion of rotation about the minor axis to the east. The low velocity resolution of our observations unfortunately prevent 


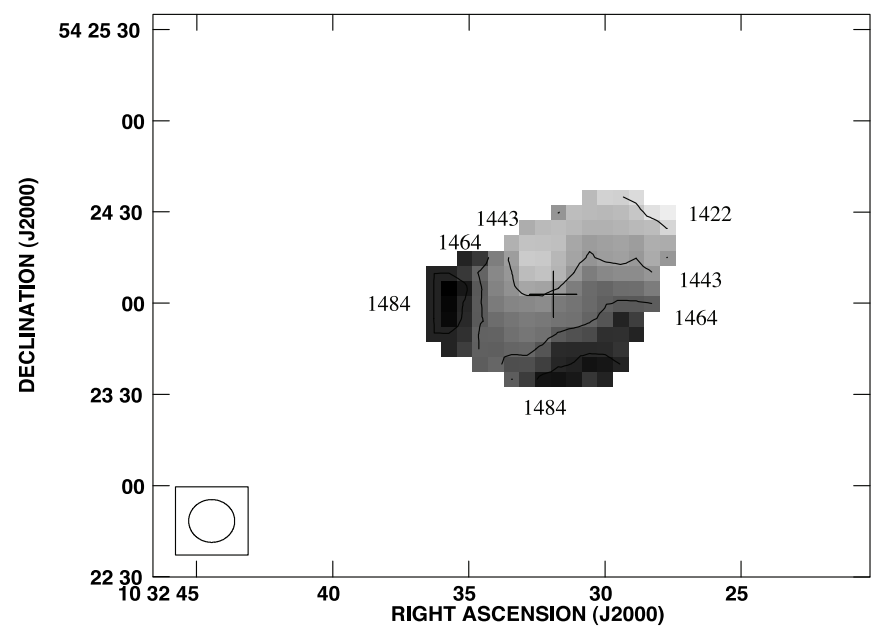

FIg. 4.- Intensity-weighted mean velocity field of Haro 2. The optical center of the galaxy is indicated with a cross; the numbers give heliocentric velocity in kilometers per second. The synthesized beam is indicated at the lower left and measures 14 ". $8 \times 14$ ". 1 .

us from confirming this latter feature. In general, the kinematics of Haro 2 inferred from its $\mathrm{H}$ I emission is consistent with the analysis of the inner $30^{\prime \prime}$ by Legrand et al. (1997) using the $\mathrm{H} \alpha$ emission line.

\subsection{The Molecular Gas in Haro 2}

Figure 5 shows the $\mathrm{CO}(1-0)$ total intensity map for Haro 2, integrated over 12 channels from 1378 to $1503 \mathrm{~km} \mathrm{~s}^{-1}$ LSR. We detect a clear arclike feature extending from southeast to northwest containing several distinct emission peaks. Taken together, these cover the same area on the sky as that in which vigorous star formation is seen in the optical, as the overlay in Figure 6 shows. There appears to be an additional concentration of gas $\sim 10^{\prime \prime}$ to the south and a possible component just as far to the northwest (labeled " $\mathrm{S}$ " and "NW" in Fig. 5); these clouds are also seen in independent data obtained with the IRAM Plateau de Bure interferometer by Fritz (2000).

After clipping the data cube at the $1 \sigma$ level, we corrected for the primary-beam response and measured the galaxy's total $\mathrm{CO}(1-0)$ flux to be $F_{\mathrm{CO}}=24.3 \pm 3.2 \mathrm{Jy} \mathrm{km} \mathrm{s}^{-1}$. Excluding the outlying concentrations of gas, we find $F_{\mathrm{CO}}=17.6 \pm 1.7 \mathrm{Jy} \mathrm{km}$ $\mathrm{s}^{-1}$. Assuming a Galactic CO-to- $\mathrm{H}_{2}$ conversion factor of $X_{\mathrm{CO}} \equiv$ $N_{\mathrm{H}_{2}} / I_{\mathrm{CO}}=2 \times 10^{20} \mathrm{~cm}^{-2}\left(\mathrm{~K} \mathrm{~km} \mathrm{~s}^{-1}\right)^{-1}$ (Strong et al. 1988; Hunter et al. 1997), we estimate the total molecular hydrogen masses following Sanders, Scoville, \& Soifer (1991):

$M_{\mathrm{H}_{2}}=1.18 \times 10^{4} M_{\odot}\left(\frac{X_{\mathrm{CO}}}{3 \times 10^{20}}\right)\left(\frac{D}{\mathrm{Mpc}}\right)^{2}\left[\frac{F_{\mathrm{CO}(1-0)}}{\mathrm{Jy} \mathrm{km} \mathrm{s}-1}\right]$.

The observed line fluxes then imply $M_{\mathrm{H}_{2}}=(7.3 \pm 0.9) \times$ $10^{7} M_{\odot}$ and $(5.3 \pm 0.5) \times 10^{7} M_{\odot}$ for all of Haro 2 and for its central regions, respectively. Multiplying by an additional factor of 1.36 to account for helium will give the total masses of gas associated with the CO-emitting structures. Use of a metallicity-dependent $X_{\mathrm{CO}}$ (Wilson 1995; Arimoto, Sofue, \& Tsujimoto 1996) would elevate these gas masses by an additional factor of 3-4 for Haro 2 's $Z \simeq 0.3 Z_{\odot}$. However, more recent studies of M33 (Rosolowsky et al. 2003) and metal-poor dwarf galaxies (e.g., Walter et al. 2004) suggest that $X_{\mathrm{CO}}$ does not depend on metallicity. Moreover, the results of

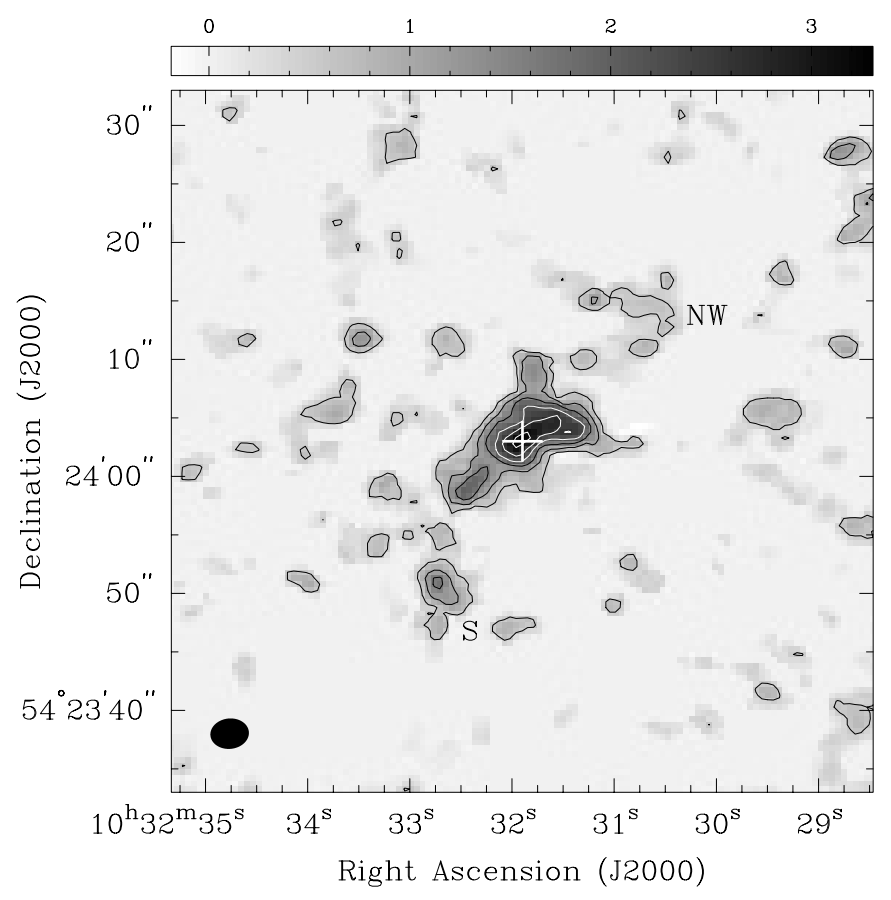

FIG. 5.-Integrated $\mathrm{CO}(1-0)$ intensity in Haro 2, with offset molecular gas concentrations south and northwest indicated. Contours are multiples of 0.52 Jy beam ${ }^{-1} \mathrm{~km} \mathrm{~s}^{-1}$ for the 3 ". $3 \times 2$ ". 6 synthesized beam shown at the lower left. Pixels with values of less than $1 \sigma$ were blanked before integrating, and the map has not been corrected for primary-beam attenuation, although the fluxes reported in the text have been. The cross marks the optical position of Haro 2 listed in Table 1.

the study of molecular gas in Haro 2 by Fritz (2000) validates the use of a nearly Galactic conversion factor to estimate $M_{\mathrm{H}_{2}}$ in this particular system.

Since interferometric data are insensitive to emission that is smoothly distributed on scales of order of that of the primary beam, we wish to estimate the fraction of the total line flux our

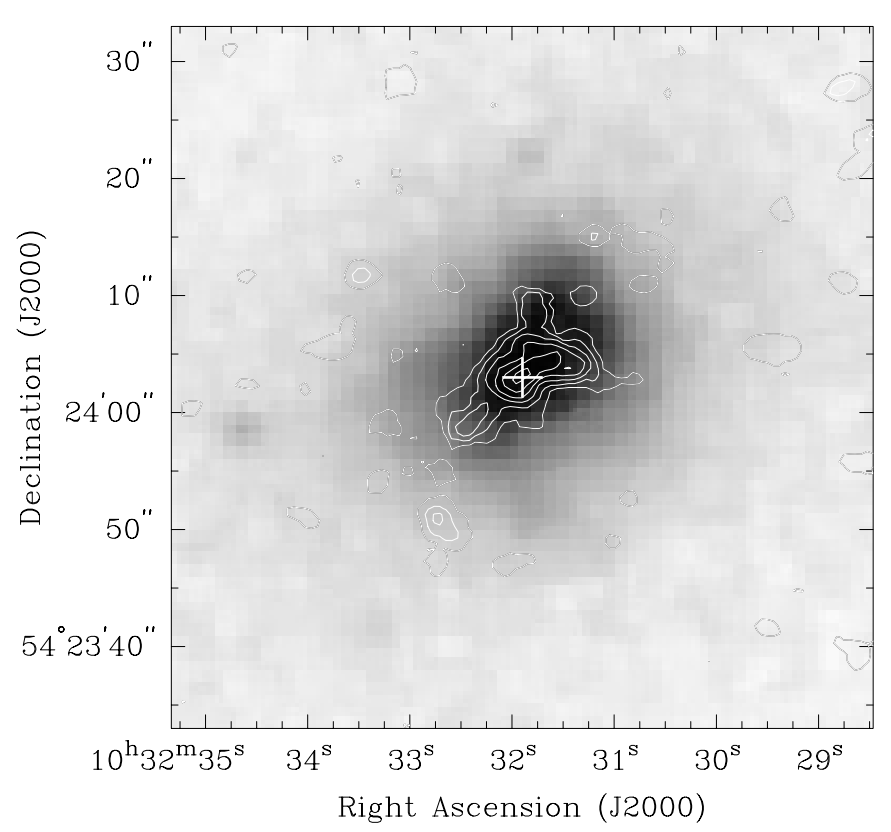

FIG. 6.-Overlay of $\mathrm{CO}(1-0)$ contours from Fig. 5 on a DSS $B$-band grayscale image. The cross marks the optical position of Haro 2 listed in Table 1. 
map recovers. We have therefore corrected the entire data cube for the primary-beam response, convolved it to the half-power beamwidths of $55^{\prime \prime}$ and $22^{\prime \prime}$ for the NRAO $12 \mathrm{~m}$ and IRAM $30 \mathrm{~m}$ telescopes, and measured the integrated intensities of our convolved data at the pointing centers used for published single-dish observations. We find we have recovered $\sim 56 \%$ of the total $\mathrm{CO}(1-0)$ emission seen by Israel et al. (1995) in their $55^{\prime \prime}$ beam and a mean (for four separate pointings) of $\sim 61 \%$ of the flux seen by Sage et al. (1992) and Barone et al. (2000) in their $22^{\prime \prime}$ beams. We make no attempt to correct for this undetected flux in what follows.

A comparison between the $\mathrm{CO}$ (gray scale) and $\mathrm{H}$ I (contours, as in Fig. 3) is shown in Figure 7, revealing interesting common features. The lower resolution obtained for the $\mathrm{H}$ I relative to the $\mathrm{CO}$ prevents a detailed comparison in the very center of Haro 2 where the CO displays a large degree of structure. In spite of this limitation, we confirm that the arclike $\mathrm{CO}$ feature follows a distribution along the same (southeastnorthwest) direction as the $\mathrm{H} \mathrm{I}$; both are coincident with the major axis of the galaxy seen in blue light. The CO is more concentrated, with a linear size smaller than the stellar component, whereas the $\mathrm{H}$ I appears more extended. With the exception of the southeast tail, the brightest $\mathrm{CO}$ regions are projected onto the innermost $\mathrm{H}$ i contour where star formation is observed to be most active (see also $\S 4$ ).

A more detailed comparison is possible between the CO map and the $\sim 3^{\prime \prime}$ resolution ROSAT HRI X-ray image published by Summers, Stevens, \& Strickland (2001). Figure 8 shows an overlay of $\mathrm{CO}$ contours on a gray-scale representation of the X-ray emission. Given that residual ROSAT pointing errors (which dominate the uncertainty in the relative astrometry of these two images) can be as large as $10^{\prime \prime}$, the agreement in the location and spatial extent of the highest surface brightness structures at both wavelengths (as well as in the optical; see Fig. 6) is quite good. In particular, there is a tantalizing alignment between the $\mathrm{CO}$ arc and the lower of the two X-ray ridges defining a tilted $\mathrm{V}$-shaped morphology in the central part of the HRI image. This coincidence can be explained if part of

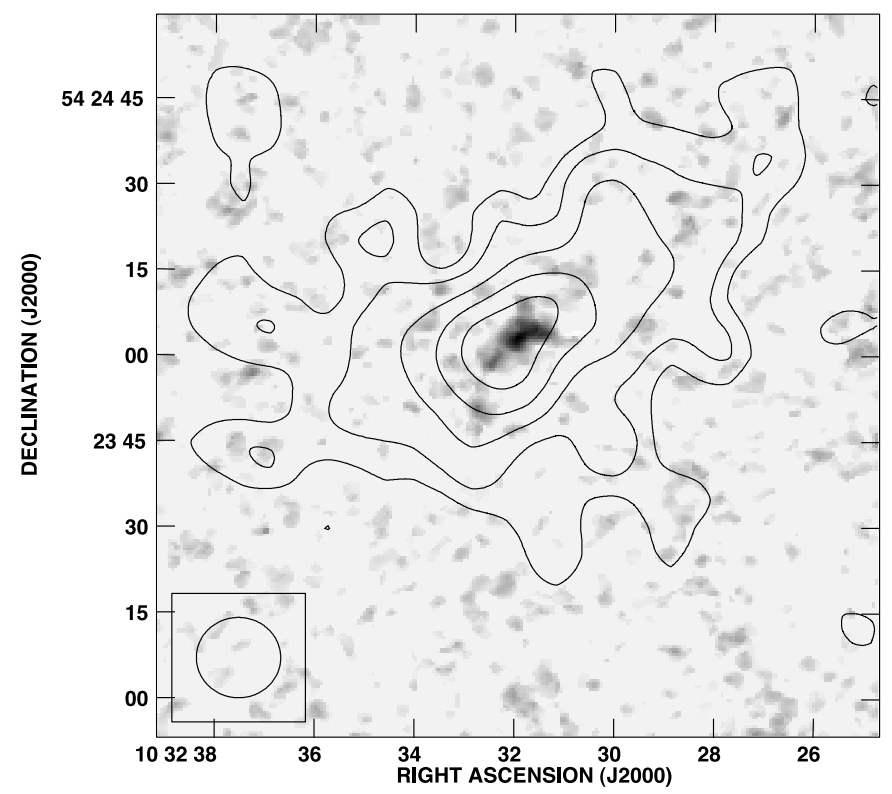

FIg. 7.-Overlay of $\mathrm{H}$ I contours from Fig. 3 on a $\mathrm{CO}(1-0)$ gray-scale representation (as in Fig. 5). The circle at the lower left indicates the size of the $\mathrm{H}$ I beam.

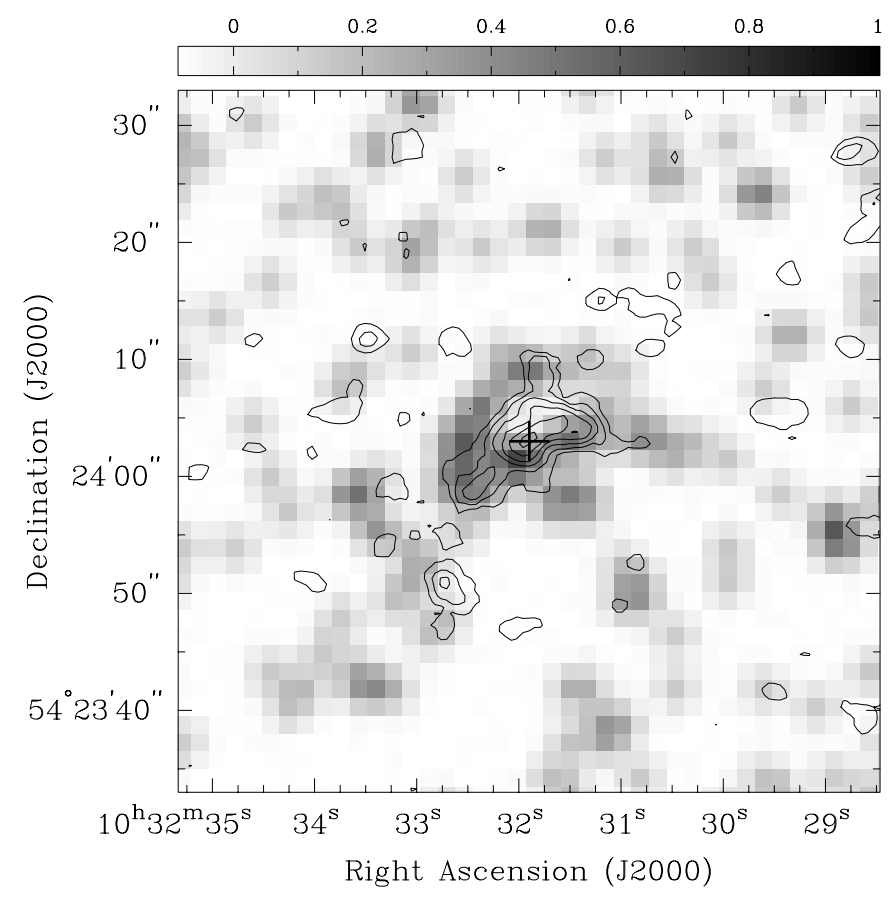

Fig. 8.-Overlay of $\mathrm{CO}(1-0)$ contours from Fig. 5 on the ROSAT HRI $\mathrm{X}$-ray gray-scale from Summers et al. (2001). The X-ray image has units of counts per pixel above background and has been smoothed by convolution with a Gaussian beam with $\sigma=3^{\prime \prime}$. The cross marks the optical position of Haro 2 listed in Table 1.

the X-ray emission is produced by shock-heated gas at the interface between an expanding superbubble and a collection of dense molecular clouds. The comparable spatial extent of $\mathrm{H} \alpha$ emission in Haro 2 (Méndez \& Esteban 2000) and evidence for an expanding shell in ionized gas kinematics (Lequeux et al. 1995; Legrand et al. 1997) offers further support for this picture. Alternatively, the X-ray emission could be a consequence of the active star formation that is taking place currently and the associated detonation of core-collapse supernovae. The formation of high-mass X-ray binaries was deemed less likely by Summers et al. (2001), based on their hardness ratio as compared with that of the extended emission seen in Haro 2. Higher resolution X-ray observations with instruments like XMM-Newton or Chandra will be needed to determine conclusively the nature of the X-ray emission and its relation to the ionized, atomic, and molecular ISM.

Figure 9 shows the channel maps for the $\mathrm{CO}(1-0)$ line in Haro 2, obtained after smoothing down the data from the original $10.4 \mathrm{~km} \mathrm{~s}^{-1}$ resolution by a factor of 3 . Although the signal-to-noise ratio for many of the features is rather meager, it is clear that the kinematics of the molecular gas is quite complex. The central arclike feature in the integrated intensity map (Fig. 5) does not appear to have a coherent velocity structure. Moreover, the spatially outlying structures do not seem to be moving at the higher relative velocities expected in the case of ordered rotation (e.g., the southeast component appears mainly in the $1426.6 \mathrm{~km} \mathrm{~s}^{-1}$ channel). As on the larger spatial scales traced by $\mathrm{H}$, the gas kinematics in the nucleus of Haro 2 is not those of a quiescent system.

\subsection{Haro 4 and Haro 26}

Figure 10 shows the $\mathrm{H}$ i channel maps for Haro 4. Despite the fact that it is almost twice as close as Haro 2, its $\mathrm{H}$ I distribution 


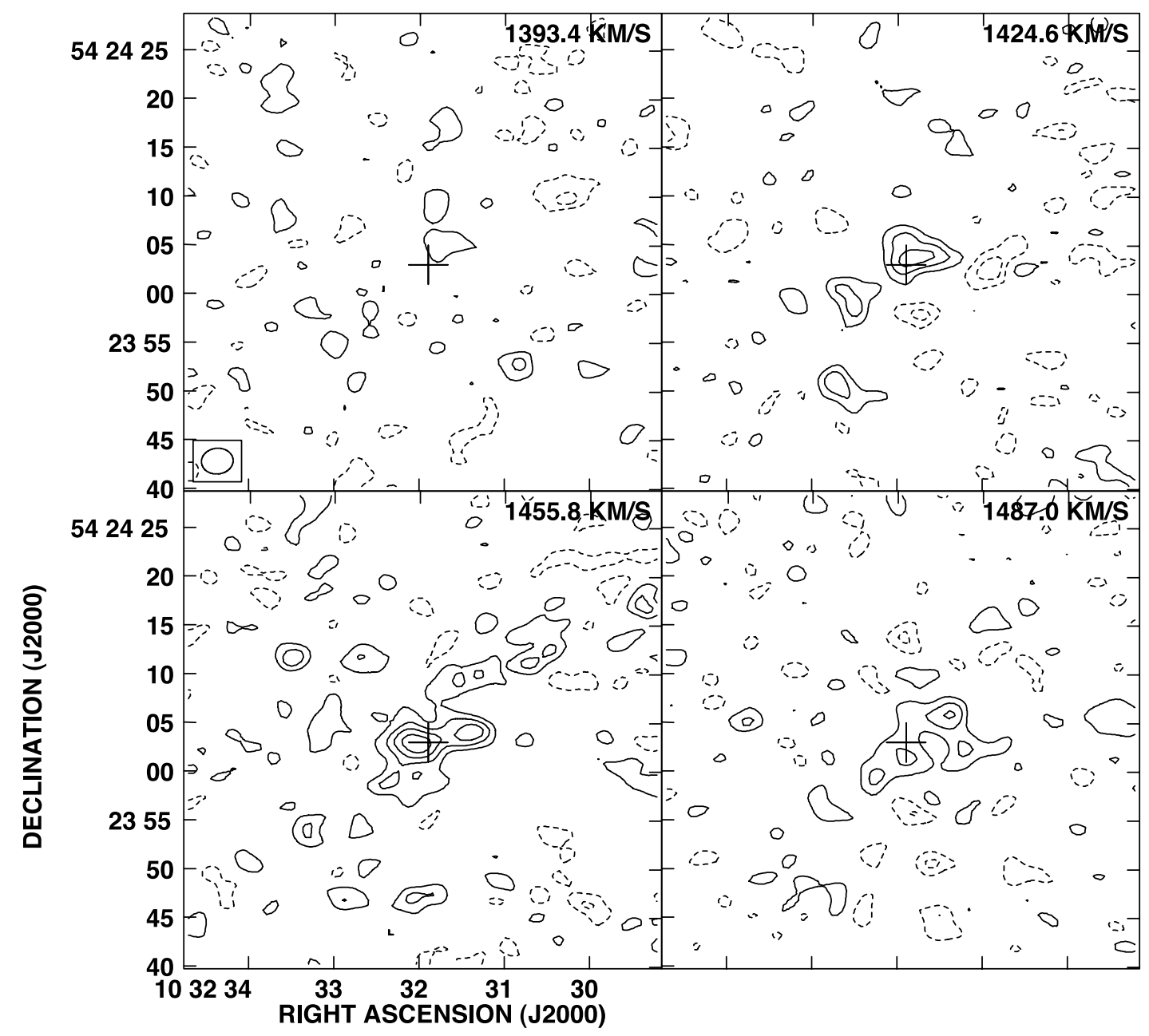

FIG. 9.- $\mathrm{CO}(1-0)$ channel maps for the velocity range integrated to produce Figs. 5 and 7 , after smoothing to $31.2 \mathrm{~km} \mathrm{~s}^{-1}$ resolution. Contours are multiples of $11 \mathrm{mJy}$ beam $^{-1}(\sim 2 \sigma)$; negative contours are dashed. The cross marks the optical position of Haro 2 (listed in Table 1); the ellipse in the top left panel shows the synthesized beam, which measures 3 " $3 \times 2$ ". 6 . These maps have not been corrected for primary-beam attenuation.

is only marginally resolved at our $14^{\prime \prime}$ resolution (equivalent to $\sim 0.5 \mathrm{kpc}$ ). The integrated $\mathrm{H}$ I map superposed on an optical DSS image is shown in Figure 11. This map confirms the highly compact nature of the $\mathrm{H} \mathrm{I}$ distribution, even though it is still almost 3 times more extended than the stellar component (see Table 6). No clear rotation pattern is detected for this galaxy, and higher resolution VLA observations are required to understand the kinematics of this object in more detail. The $\mathrm{H}_{\mathrm{I}}$ content we derive for this galaxy $\left(M_{\mathrm{H}_{\mathrm{I}}}=0.19 \times 10^{8} M_{\odot}\right)$ agrees with the single-dish value reported by Gordon \& Gottesman (1981), confirming that no $\mathrm{H}$ I emission is missed by the VLA.

As mentioned in $\S 2.2$, no $\mathrm{CO}(2-1)$ emission was detected from Haro 4. From the per-channel rms of $9 \mathrm{mK}\left(T_{A}^{*}\right)$, the corresponding conversion factor $50 \mathrm{Jy} \mathrm{K}^{-1}$ for the CSO at $230 \mathrm{GHz}$ (Gerin \& Phillips 2000), and an assumed velocity width of $42 \mathrm{~km} \mathrm{~s}^{-1}$ (matching the H I FWHM), we derive a $3 \sigma$ upper limit on the integrated $\mathrm{CO}(2-1)$ flux from Haro 4 of $\leq 21$ Jy $\mathrm{km} \mathrm{s}^{-1}$. If the $\mathrm{CO}(2-1) / \mathrm{CO}(1-0)$ intensity ratio is $\sim 0.5$ in temperature units, as is the case for the two BCDs (Haro 2 and Mrk 297) mapped by Sage et al. (1992), then we would expect the $\mathrm{CO}(1-0)$ line flux from Haro 4 to be $\leq 43 \mathrm{Jy} \mathrm{km} \mathrm{s}^{-1}$. Equation (1) then implies that Haro 4 has a molecular hydrogen mass $M_{\mathrm{H}_{2}} \leq 1.6 \times 10^{7} M_{\odot}$. Correction of $X_{\mathrm{CO}}$ for the possible dependence on metallicity according to the prescriptions of Wilson (1995) and Arimoto et al. (1996) could increase this upper limit on $M_{\mathrm{H}_{2}}$ by factors of 8 and 16 , respectively.

Projected some $22^{\prime}$ southwest of Haro 4 , the neighboring spiral galaxy Haro 26 is well resolved by the VLA beam and spans a much larger range in velocity. The channel maps are presented in Figure 12. This object is a clear example of a galaxy in differential rotation seen almost edge-on. H I emission extends from roughly 600 to $800 \mathrm{~km} \mathrm{~s}^{-1}$. Figure 13 shows a composite plot of integrated $\mathrm{H}$ I profiles of Haro 4 and Haro 26. The total $\mathrm{H}$ I surface brightness map presented in Figure 14 shows the integrated $\mathrm{H}_{\mathrm{I}}$ emission of Haro 26 superposed on a DSS image. As is common, the $\mathrm{H}$ I extends well beyond the optical image, in this case by about a factor of 1.5 (see Table 6). We measure a total $\mathrm{H}$ I flux for Haro 26 of $40 \mathrm{Jy} \mathrm{km} \mathrm{s}{ }^{-1}$, in good agreement with previous $\mathrm{H}$ I line observations. Tifft \& Cocke (1988) observed this galaxy with the $91 \mathrm{~m}$ NRAO telescope and obtained an $\mathrm{H}$ I flux of $48.3 \mathrm{Jy} \mathrm{km} \mathrm{s}{ }^{-1}$, very close to the $49.4 \mathrm{Jy} \mathrm{km} \mathrm{s}^{-1}$ reported by Haynes et al. (1999). More recently, Taylor et al. (1994) observed this galaxy with the VLA and reported an $\mathrm{H}$ i mass of $10.1 \times 10^{8} M_{\odot}$ (scaled to the our assumed distance of $7.9 \mathrm{Mpc}$ ), only $15 \%$ larger than the value of $8.5 \times 10^{8} M_{\odot}$ that we report in this work (see Table 5). 


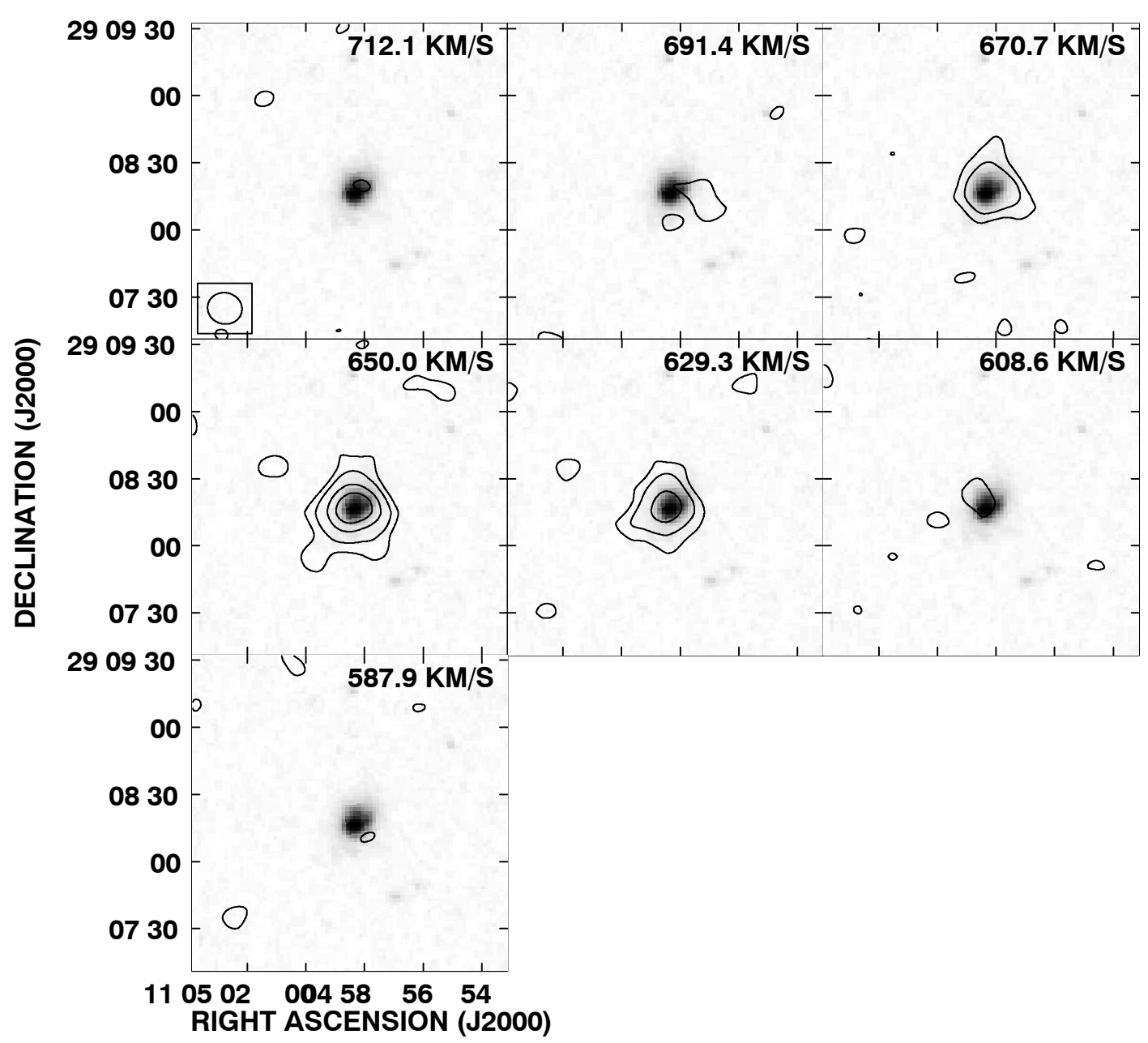

FIg. 10.-Mosaic of the $\mathrm{H}$ I line emission channels of Haro 4 overlaid on a DSS $B$-band image. Contours are $-2.5,2.5,5.0,10$, and $15 \sigma$; negative contours are dashed. The beam size indicated in the top left panel is $15^{\prime \prime} 5 \times 14$ ". 0 .

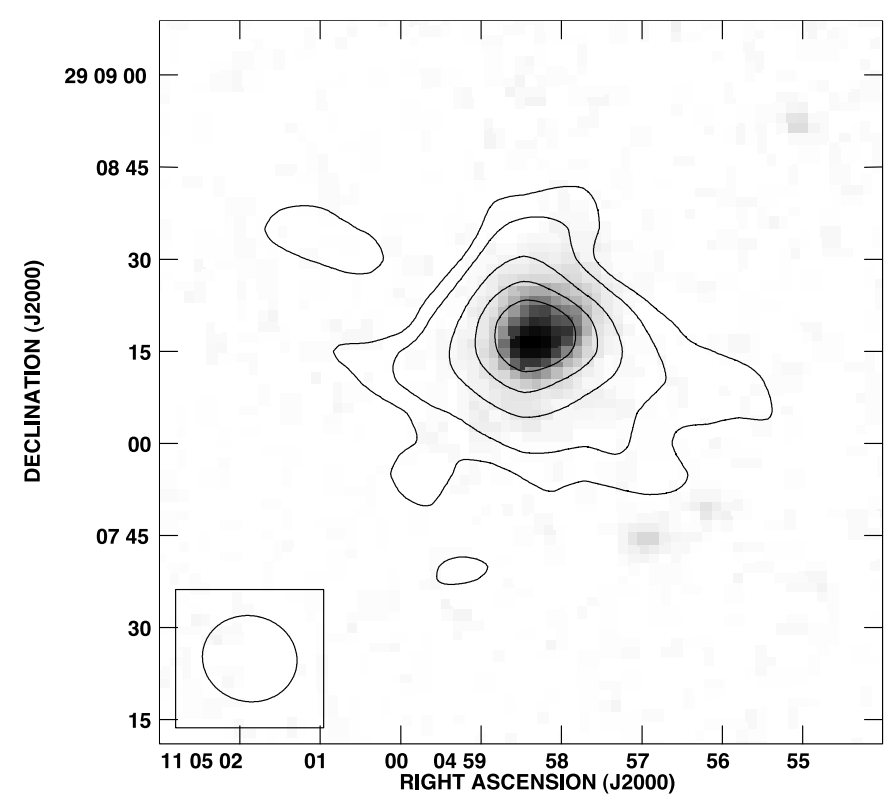

FIG. 11.-H I surface brightness contours for Haro 4, superposed on a DSS $B$-band gray-scale image. The contours are $2.1(5 \sigma), 6.2,12.3$, and $18.5 \times$ $10^{20} \mathrm{~cm}^{-2}$. The beam size is $15^{\prime \prime} .5 \times 14^{\prime \prime} .0$.
Figure 15 shows both Haro 4 and Haro 26 in a single map at larger scale. Their projected separation is $\sim 45 \mathrm{kpc}$. The galaxy seen on the DSS image midway between Haro 4 and Haro 26 is CGCG 155-052, a background galaxy at $z \sim 0.03$. The conspicuous H I extension to the northeast of Haro 26, previously reported by Taylor et al. (1994), is contiguous with the rest of the galaxy in the velocity field (Fig. 16). The galaxy appears symmetrical only out to a radius of $\sim 1^{\prime}$ (some $2.3 \mathrm{kpc}$ ), beyond which the northern tip veers northeast toward Haro 4. The radio continuum map of Haro 26 (Fig. 17) shows emission coinciding with this northeast $\mathrm{H}$ i feature. To the south isolated radio continuum blobs extend farther than the $\mathrm{H}$, with intensities at the $\sim 2.5 \sigma$ level.

\section{DISCUSSION}

\subsection{Haro 2}

Haro 2 is a peculiar member of the BCD class. Most BCDs are gas-rich, dwarf irregular systems manifesting bursts of star formation. As first pointed out by Loose \& Thuan (1986), however, Haro 2 has an $R^{1 / 4}$ rather than exponential surface brightness profile in the optical (see also Cairós et al. 2001), leading those authors to classify Haro 2 as a dwarf elliptical galaxy. Haro 2 has a small $\mathrm{H}$ I-to-optical diameter ratio, 
TABLE 6

Inferred Global Parameters

\begin{tabular}{|c|c|c|c|c|c|c|c|}
\hline ID & $D_{\mathrm{H}_{\mathrm{I}}} / D_{\mathrm{opt}}$ & $\begin{array}{c}M_{\mathrm{H}} / L_{B} \\
\left(M_{\odot} / L_{\odot}\right)\end{array}$ & $\begin{array}{c}M_{\text {dyn }} \\
\left(10^{9} M_{\odot}\right)\end{array}$ & $\begin{array}{l}M_{\text {dyn }} / L_{B} \\
\left(M_{\odot} / L_{\odot}\right)\end{array}$ & $M_{\mathrm{H}_{\mathrm{I}}} / M_{\mathrm{dyn}}$ & $\begin{array}{c}M_{\mathrm{H}_{2}} \\
\left(10^{7} M_{\odot}\right)\end{array}$ & $M_{\mathrm{H} \mathrm{I}} / M_{\mathrm{H}_{2}}$ \\
\hline Haro $2 \ldots \ldots$. & 1.4 & 0.09 & 2.5 & 1.0 & 0.09 & 7.3 & 3.1 \\
\hline Haro 4 ........ & 2.8 & 0.17 & 0.5 & 4.8 & 0.03 & $\leq 1.6$ & $\geq 1.2$ \\
\hline Haro $26 \ldots . .$. & 1.5 & 0.76 & 11.5 & 10.3 & 0.07 & $\ldots$ & $\ldots$ \\
\hline
\end{tabular}

$D_{\mathrm{H}} / D_{\text {opt }} \sim 1.4$, whereas the $\mathrm{H}$ I distributions of most BCDs are usually considerably larger than their optical disks $\left(D_{\mathrm{H}} / D_{\text {opt }} \sim 2-5\right.$ : Taylor et al. 1994; van Zee et al. 1998; van Zee, Salzer, \& Skillman 2001). The H I content of Haro 2 is normal relative to other BCDs, in contrast to its $M_{\mathrm{H}_{\mathrm{I}}} / L_{B}$ ratio (see Table 6), which is at the low end for BCDs (Smoker et al. 2000; Lee et al. 2002; Salzer et al. 2002). Of the sample studied by van Zee et al. (1998, 2001), Haro 2 is most similar to UM 38 and Mrk 324, two other dwarf elliptical galaxies (Doublier et al. 1997; Cairós et al. 2001) with comparable atomic gas properties. UM 465 resembles Haro 2 in that it is a dwarf elliptical galaxy detected in both $\mathrm{CO}$ and $\mathrm{H}$ I emission, although it has a somewhat smaller $\mathrm{H}$ I mass and somewhat larger metallicity (Sage et al. 1992).

While Haro 2 does resemble a BCD in terms of its optical morphology and gas content, it is almost unique in the kinematics of its neutral atomic and molecular gas. Whereas in disk-dominated systems the kinematic and isophotal major axes are aligned, in the case of Haro 2 they are nearly perpendicular. One of the few dwarf galaxies with equally pathological kinematics is NGC 5253, whose own molecular and atomic gas components also appear to be predominantly rotating about the galaxy's major axis (Kobulnicky \& Skillman 1995; Meier et al. 2002). This configuration has been postulated to result from a tidal interaction, or from the accretion of gas from a formerly gas-rich (now stripped) companion. Its kinematic complexity, together with the presence of a dust lane (like that seen in Haro 2; Möllenhoff, Hummel, \& Bender 1992), suggest NGC 5253 may be a dwarf version of a giant, postinteraction, dust lane elliptical galaxy (Oosterloo et al. 2002). In the context of this interaction scenario some of the similarities and differences between Haro 2 and NGC 5253 deserve special attention.

In terms of star formation NGC 5253 appears to be undergoing a burst that is markedly younger and more intense than that in Haro 2. While the youngest stars in Haro 2 have ages $\sim 10$ Myr (Fanelli, O’Connell, \& Thuan 1988), population synthesis fits to star clusters in NGC 5253 suggest ages in the 1-8 Myr range (Tremonti et al. 2001), and its extremely luminous and deeply embedded "supernebula" (Turner, Beck, \& Ho 2000; Gorjian, Turner, \& Beck 2001; Turner et al. 2003) must be younger still. Haro 2's older burst age is also indicated by its lower millimeter/centimeter continuum flux ratio. In particular, our $3 \sigma$ upper limit of $2.3 \mathrm{mJy}$ on $112 \mathrm{GHz}$ emission from Haro 2 (see $\S 2.2$ above) implies that no more than $3.5 \mathrm{mJy}$ of its $24.6 \mathrm{mJy}$ observed at $1.4 \mathrm{GHz}$ can be due to optically thin free-free emission (assuming a spectral index $\alpha=-0.1$ ). Virtually all of the $1.4 \mathrm{GHz}$ flux density from NGC 5253, on the other hand, is thermal (Turner, Ho, \& Beck 1998; Meier et al. 2002). Finally, the star formation efficiency in NGC 5253 is considerably higher, with its $L_{\mathrm{IR}} / M_{\mathrm{H}_{2}} \simeq 800 L_{\odot} / M_{\odot}$ (Turner et al. 1997) exceeding the more modest $\sim 50 L_{\odot} / M_{\odot}$ we infer for Haro 2 (see $\S 4.3$ below).
It is tempting to associate the older, less vigorous starburst in Haro 2 with a dynamical disturbance that occurred longer in the past than the one currently afflicting NGC 5253. Several lines of evidence indeed favor this interpretation. First, much of the molecular gas in the vicinity of NGC 5253 appears still to be falling inward and is not associated with the regions of most intense star formation (Meier et al. 2002). Haro 2, in contrast, has a $\mathrm{CO}(1-0)$ distribution whose spatial extent broadly

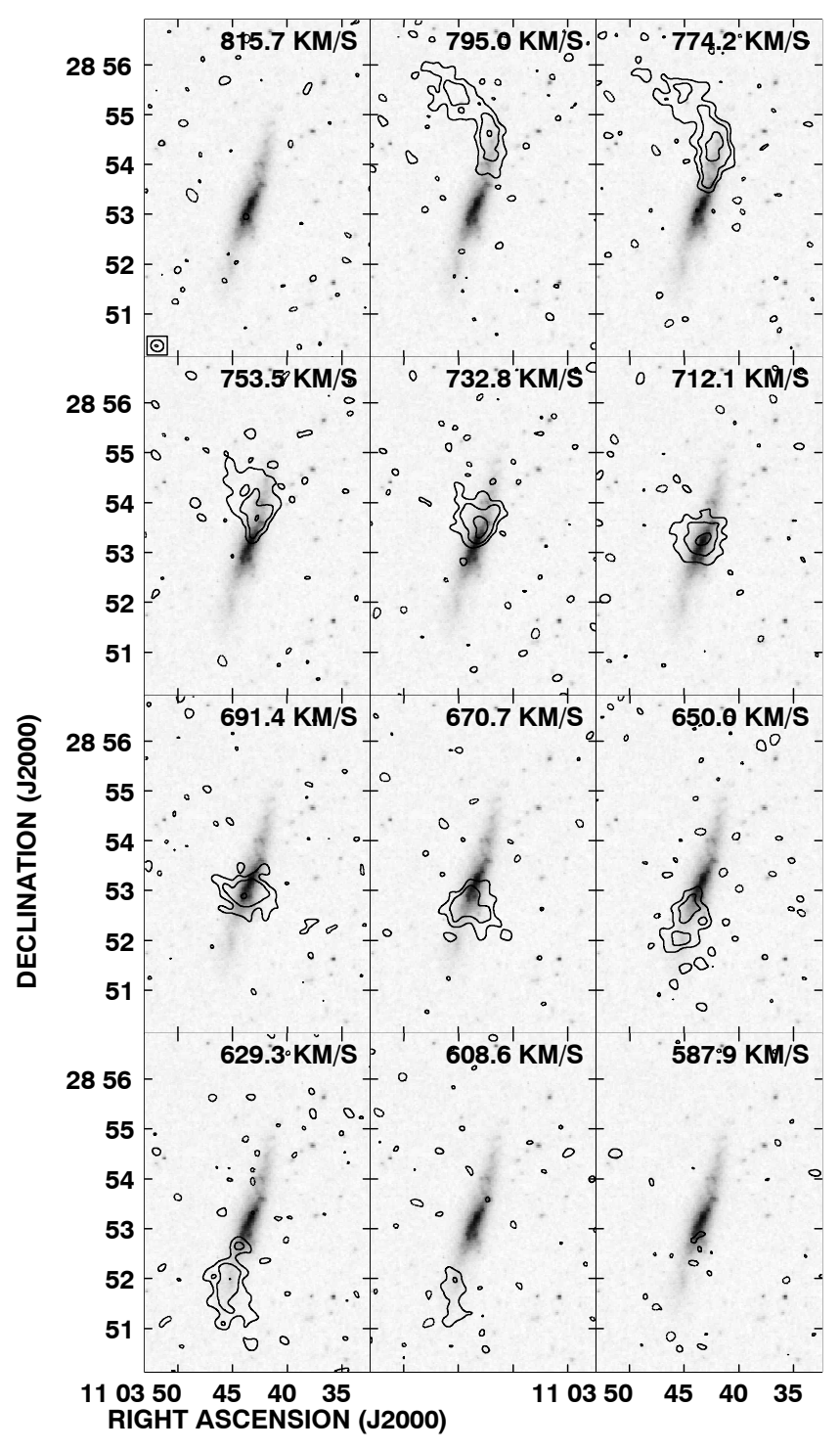

FIG. 12.-Mosaic of the channels showing $\mathrm{H}$ I line emission from Haro 26, superposed on a DSS $B$-band gray-scale image. Contours are $-2.5,2.5,5.0$, and $10 \sigma$; negative contours are dashed. The beam size, indicated in the top left panel, is $15^{\prime \prime} .5 \times 14^{\prime \prime} .0$. 


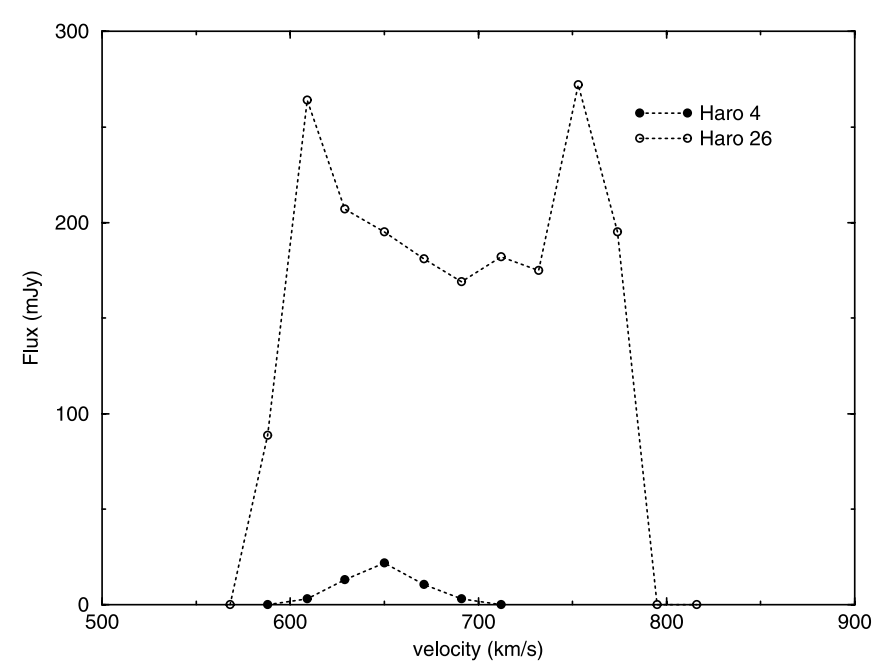

Fig. 13.-Global $\mathrm{H}$ I profile for Haro 4 and Haro 26, obtained by integrating the channel maps over the areas of the sources after continuum subtraction, blanking, and correction for primary-beam attenuation.

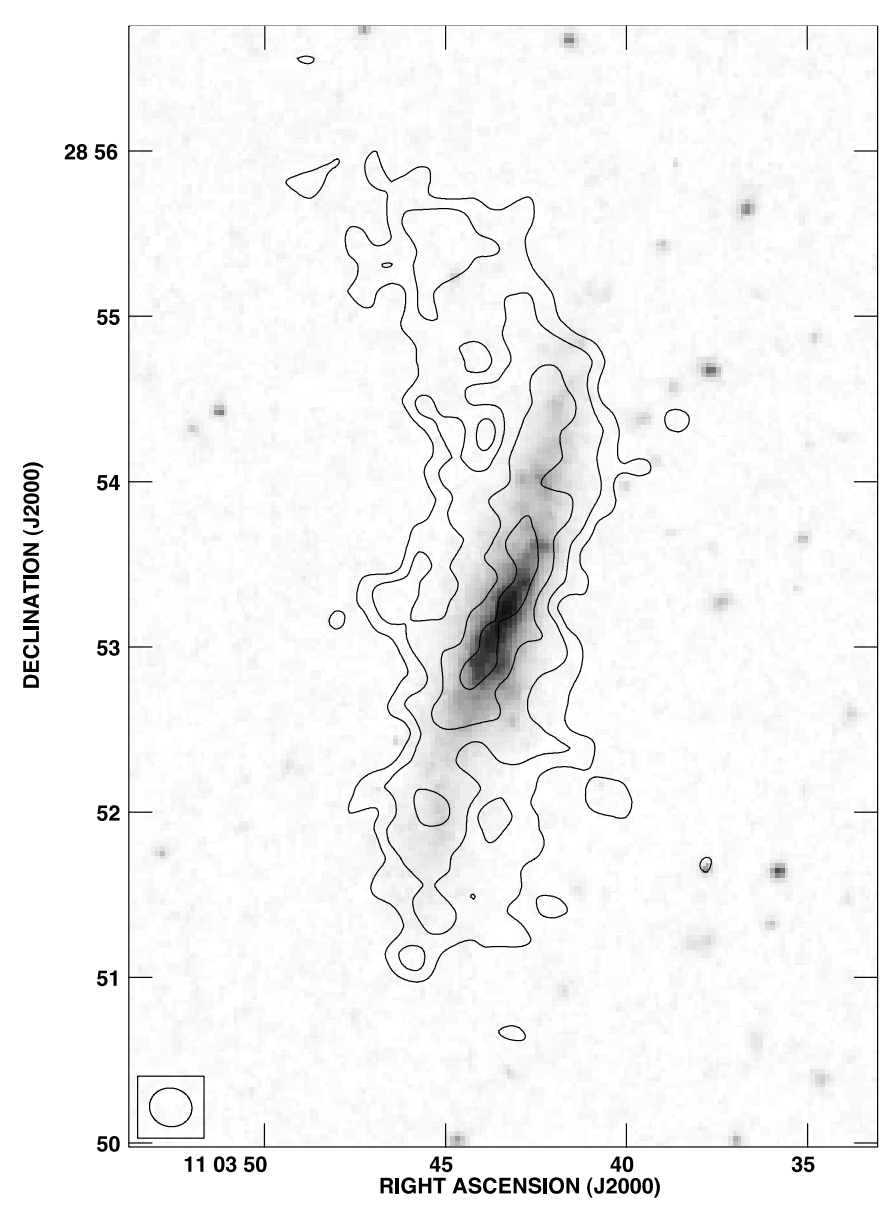

FIG. 14.- - H I surface density contours for Haro 26, superposed on a DSS $B$-band gray-scale image. The contours are $2.1(5 \sigma), 6.2,12.3$, and $18.5 \times 10^{20} \mathrm{~cm}^{-2}$. The beam measures $15^{\prime \prime} .5 \times 14^{\prime \prime} .0$.

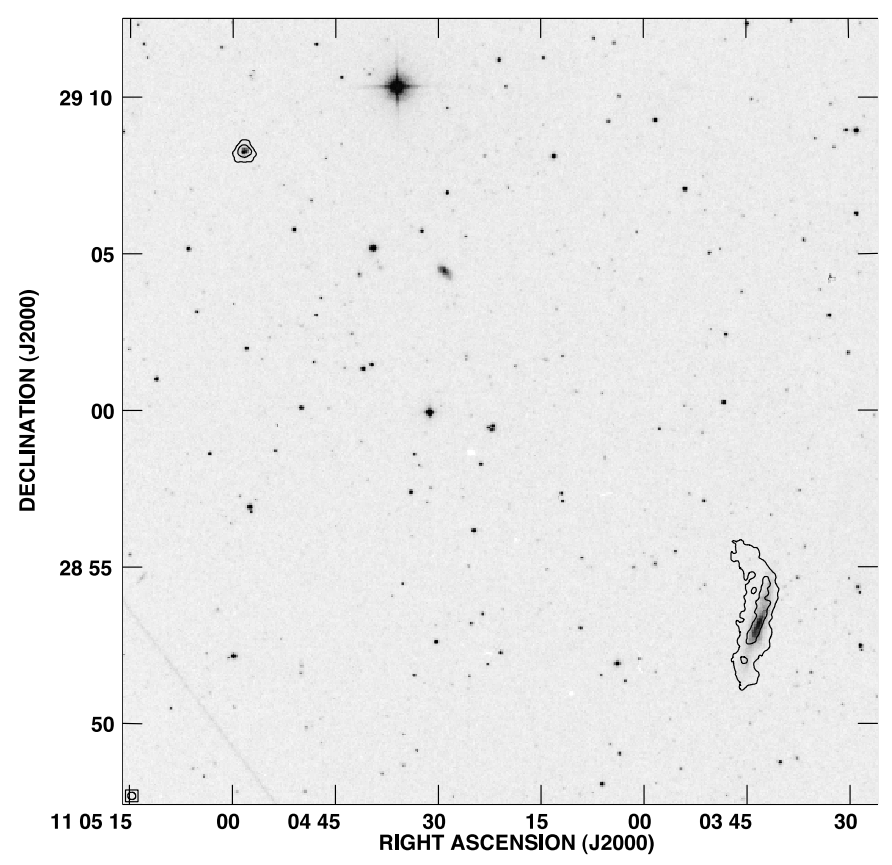

FIG. 15.- - Larger scale $\mathrm{H}$ I map of the field including Haro 4 and Haro 26 (at upper left and lower right, respectively), overlaid on a DSS $B$-band grayscale image. For clarity, only the two lowest contours from Figs. 11 and 14 are shown.

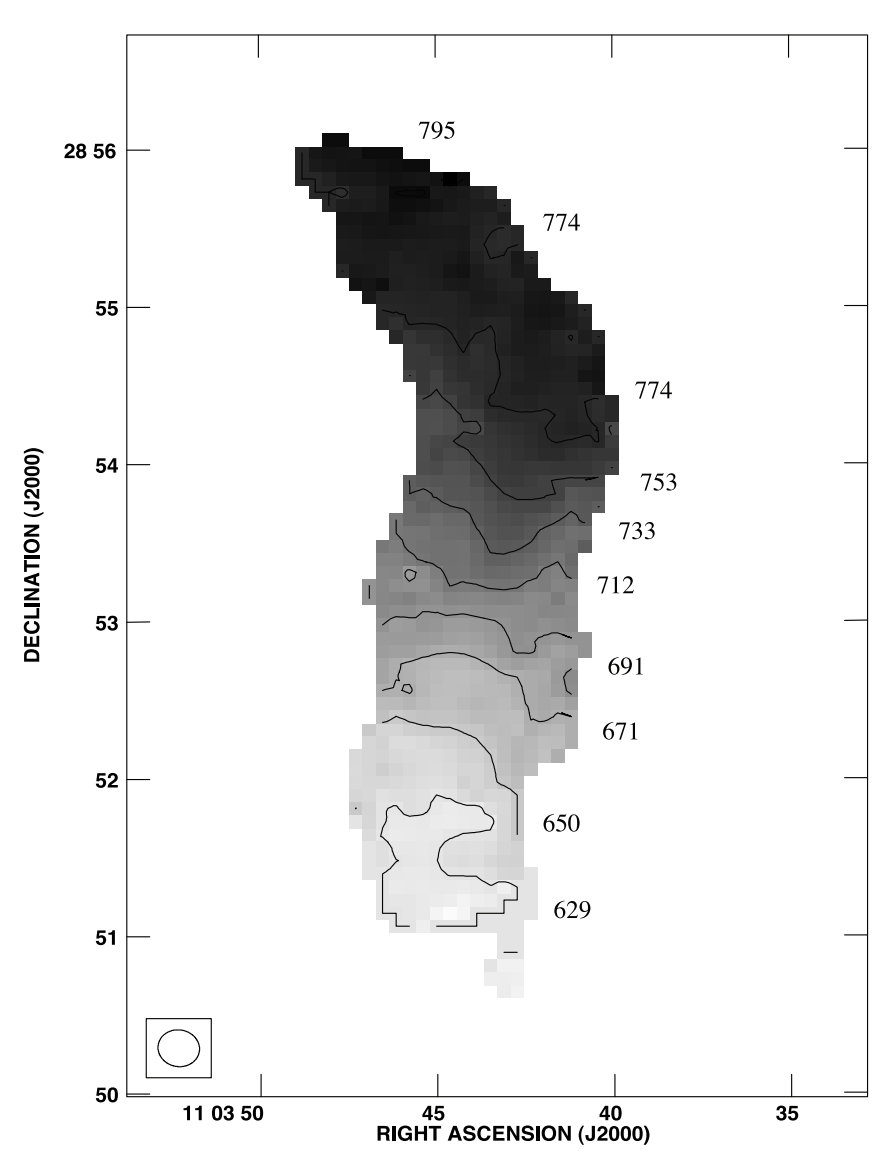

FIG. 16.- Intensity-weighted mean velocity field of Haro 26. The numbers indicate heliocentric velocity in kilometers per second. The beam size is indicated by the ellipse and measures 15 " $5 \times 14 . " 0$. 


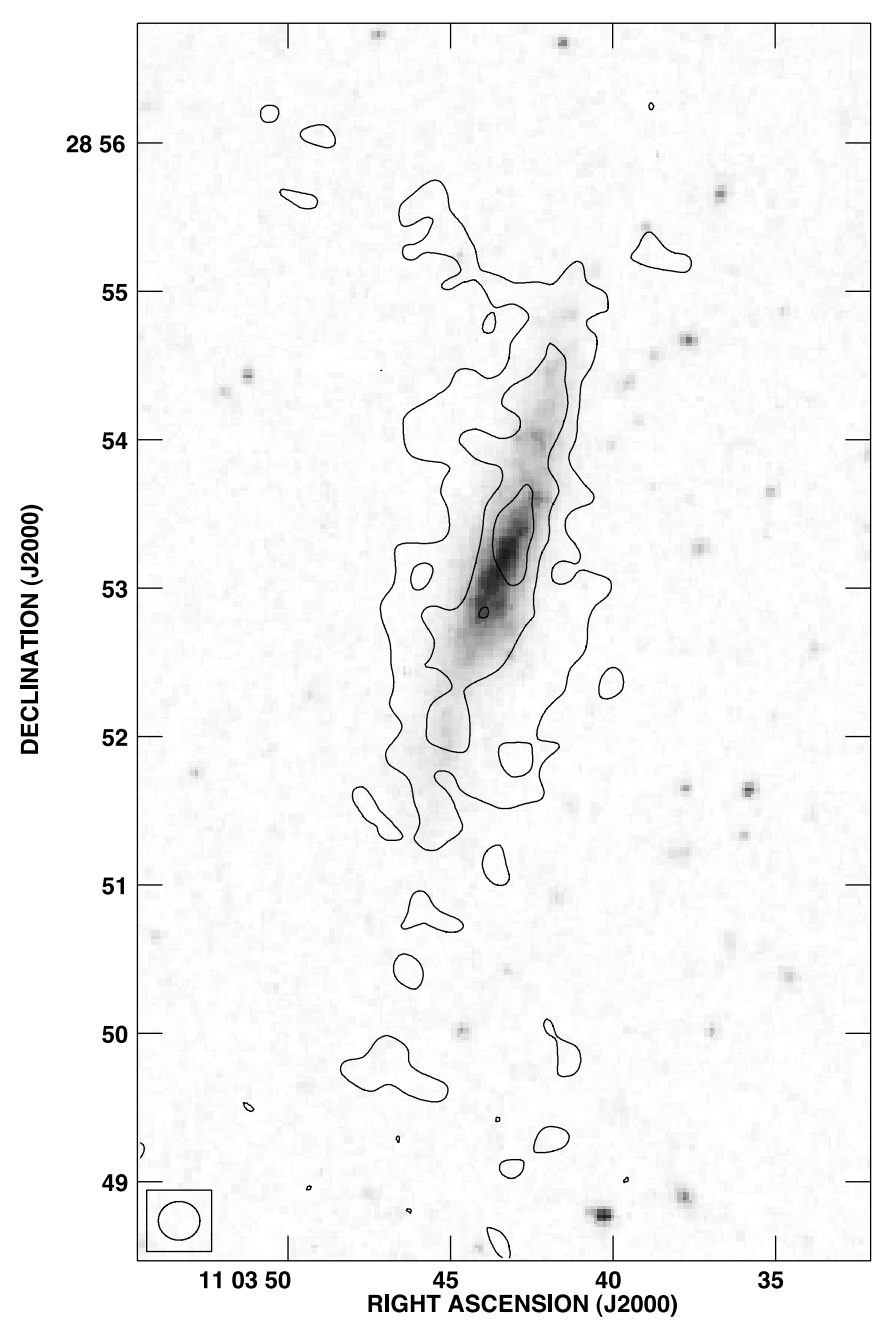

FIG. 17.-Radio continuum emission for Haro 26, obtained by averaging the line-free channels of the $\mathrm{H}_{\mathrm{I}}$ data cube. Contours are 2.5, 5.0, and $7.5 \sigma$ ( $1 \sigma \sim 0.1 \mathrm{mJy}$ beam $^{-1}$ ) and are superposed on a DSS $B$-band gray-scale image. The beam is indicated in the lower left corner and measures $16^{\prime \prime} 8 \times 15^{\prime \prime} .6$.

matches that of its star formation as traced by $\mathrm{H} \alpha$, X-ray, and optical continuum emission; this agreement suggests that Haro 2's molecular component has finished coalescing toward the bottom of its potential well. Second, the disagreement between the position angles of the $\mathrm{H}$ I and optical disks in NGC 5253 - in fact, they are nearly perpendicular on the skyrepresents a level of disturbance not seen in Haro 2, where the $\mathrm{H}$ I follows the optical disk quite well. Third, we recall from $\S 2.1$ that our VLA data failed to recover about half of the $\mathrm{H}$ I emission detected in single-dish observations of Haro 2 (see Fig. 2). Diffuse, extended emission could very well surround the galaxy on $\sim 10^{\prime}$ scales, having accumulated there in the same way that late infall in more massive postinteraction systems can build regular $\mathrm{H}$ I disks (Oosterloo et al. 2002).

Considering all of the above evidence, we conclude that Haro 2 is most likely a somewhat older cousin of NGC 5253a dwarf elliptical galaxy that has recently captured gas in a close interaction or merger with an initially gas-rich companion. For Haro 2 an obvious candidate donor is the putative companion lying 4.'7 to the east (Huchtmeier et al. 1995), whose nondetection in $\mathrm{H}$ i by those authors could be explained if it lost all its gas in the interaction. If at the same redshift, its projected separation from Haro 2 would be a mere $27 \mathrm{kpc}$; however, there is no redshift estimate for this object, and the real distance that separates it from Haro 2 remains uncertain. Alternatively, Pustilnik et al. (2001b) propose the galaxy UGC 5676 as the trigger of the burst of star formation. This object lies much further away in projection, some $36^{\prime}$ ( $\sim 200 \mathrm{kpc}$ ) northeast of Haro 2, but is known to share roughly the same redshift. Both neighbors are fainter than Haro 2 (by 3 and $1.6 \mathrm{mag}$, respectively) and presumably less massive as well, consistent with our assumption that one of them would have lost its gas mass to Haro 2 rather than the other way around. In view of the mass-metallicity relation for dwarf galaxies (e.g., Bender, Burstein, \& Faber 1992), Haro 2 should have received an infusion of less enriched gas from its less massive companion. This would tend to have left Haro 2 with a lower metallicity in its interstellar medium than in its stars, although exactly how much lower is difficult to predict without knowing what fraction of its gas mass originated elsewhere. We note that the possibility of tidal interaction with more than one object at different times should also not be ruled out.

In the "passing encounter" scenario depicted above-and even if Haro 2 is actually in the final stages of a merger with a gas-rich dwarf-gas would have been captured at a random angle with respect to the major axis of Haro 2 and would have since settled into a relatively stable orbit encircling the galaxy's long axis. The fraction of gas settling further into the gravitational potential of Haro 2 would accumulate and reach densities high enough for molecules to form. Molecular gas spiralling inward would naturally shock, leading to the current burst of star formation we are witnessing.

Even if the $\mathrm{H}$ I gas is of relatively recent acquisition, its motion is by now dominated by the gravitational potential of Haro 2, any plausible donor having passed perigalacticon and no longer exerting any tidal pull. If we assume that the object $27 \mathrm{kpc}$ to the east was the source of the accreted gas, and if we take as an order of magnitude estimate a relative velocity between the two objects of $150 \mathrm{~km} \mathrm{~s}^{-1}$ (a reasonable upper limit, according to Taylor et al. 1994), 180 Myr have elapsed since closest approach, i.e., a few revolutions of the captured gas in the potential of Haro 2. It is therefore valid as a first approximation to assume for dynamical mass estimates that the gas is moving in roughly circular orbits. We recall that the $\mathrm{H}$ I and $\mathrm{CO}$ velocity fields show that any rotation is within a plane more or less perpendicular to the optical major axis. Given that no value for the inclination is known, we can only estimate a lower limit to the dynamical mass. The extent of the gas along the kinematical major axis is $\sim 1.0$. Taking a distance to Haro 2 of $19.5 \mathrm{Mpc}$ and a projected rotational velocity of $62.5 \mathrm{~km} \mathrm{~s}^{-1}$, we find a value of $M_{\mathrm{dyn}}=2.6 \times 10^{9} M_{\odot}$ and an $M_{\text {dyn }} / L_{B}$ ratio $=1.0$. Even when taking into account the fact that the dynamical mass estimate is a lower limit, for any reasonable $M / L$ ratio for the stars in Haro 2 there does not seem to be a need to invoke any dark matter. This is contrary to what is generally seen in BCDs, which, if anything, are more dark matter dominated than large, spiral galaxies.

Our estimate of $180 \mathrm{Myr}$ as the time of closest approach leaves ample time for the $\mathrm{H}$ I to turn into $\mathrm{H}_{2}$. Following the same arguments as given in Braine et al. (2001), the time to transform $20 \%$ of atomic hydrogen into $\mathrm{H}_{2}$ is $t_{20} \% \approx \frac{10^{7}}{n_{\mathrm{H}_{1}}} \mathrm{yr}$, where $n_{\mathrm{H}_{\mathrm{I}}}$ is the $\mathrm{H}$ I volume density (Hollenbach, Werner, \& Salpeter 1971 and references therein). A rough estimate of the latter can be made by taking the average of the $\mathrm{H}$ I column density across the area where $\mathrm{CO}$ has been detected and assuming a line-of-sight thickness for the $\mathrm{H}$ i of order $200 \mathrm{pc}$. 
The $\mathrm{H}$ I volume density thus derived is $n_{\mathrm{H}_{\mathrm{I}}} \approx 3 \mathrm{~cm}^{-3}$, suggesting that the time needed to transform a substantial amount of atomic to molecular gas is less than $10^{7} \mathrm{yr}$, much shorter than the typical time for solar-mass stars to form subsequently $(\approx 30 \mathrm{Myr})$. The value $t_{20 \%}$ is an appropriate indicator for the timescale on which molecular gas forms in Haro 2, as most of the gas is still atomic. Lower metallicities and hence lower dust content will decrease linearly the rate at which this occurs.

The prominent nuclear star formation in Haro 2 has been analyzed in the UV and optical by Lequeux et al. (1995) using HST. These authors find evidence for gas flowing away from the central $\mathrm{H}$ II region at a velocity of $200 \mathrm{~km} \mathrm{~s}^{-1}$, notably strong Ly $\alpha$ emission with a clear $\mathrm{P}$ Cygni profile and a complex velocity structure (see also Legrand et al. 1997). The column density of neutral hydrogen in this outflowing gas is $N(\mathrm{H}) \simeq 7 \times 10^{19} \mathrm{~cm}^{-2}$. This is only a small fraction of the column density measured in the VLA map, which is of order $2 \times 10^{21} \mathrm{~cm}^{-2}$ (averaged over the central pixel at a spatial resolution of $\sim 1.4 \mathrm{kpc}$ ). The data are best explained by assuming that the central starburst has created an expanding supergiant shell measuring $\sim 1.8 \mathrm{kpc}$ and expanding at $200 \mathrm{~km}$ $\mathrm{s}^{-1}$. In this model the outflowing gas is predominantly ionized by the central star cluster, and the total mass involved in the outflow (if distributed in a spherical shell immediately surrounding the $\mathrm{H}$ II region) is of order $10^{7} M_{\odot}$. Unfortunately, the size of this proposed shell is too close to the resolution limit of the $\mathrm{H}$ i observations for us to be able to detect its signature in our data cube.

\subsection{Haro 4 and Haro 26}

Haro 4, shown in Figure 11, appears to be highly centrally concentrated in both its $\mathrm{H}$ I and optical light distributions. The $\mathrm{H}$ I mass of this galaxy is low compared with that of most BCDs previously observed in H I (van Zee et al. 1998, 2001; Pustilnik et al. 2001b); we estimate a lower limit for its dynamical mass of $M_{\mathrm{dyn}}=5 \times 10^{8} M_{\odot}$. However, as no clear indications for rotation are seen, this should only be considered indicative. Other parameters of Haro 4 are rather typical among BCDs, such as its $M_{\mathrm{H}} / L_{\mathrm{B}}$ and $D_{\mathrm{H}_{\mathrm{I}}} / D_{\text {opt }}$ ratios. Kunth et al. (1998) find in Haro 4 a broad damped Ly $\alpha$ absorption profile centered at the wavelength corresponding to the redshift of the $\mathrm{H} \alpha$-emitting gas. They show that this occurs chiefly because the $\mathrm{H}$ I gas is smooth and virtually static, as confirmed by the new VLA observations, with respect to the ionized region where $\operatorname{Ly} \alpha$ photons originate.

For Haro 26, the H I map in Figure 14 confirms the strong warping toward the northeast already observed in the optical (Sandage \& Bedke 1994) and in H I (Taylor et al. 1994). While the former authors find no nearby companion to explain the warp, Taylor et al. (1994) suggest that the emission extending toward the northeast may in fact be a separate object. We find no evidence to support this scenario, however, as our velocity field in the northeast extension appears to be continuous with that of the main galaxy. From the channel maps (Fig. 12) and the zeroth- and first-moment maps (Figs. 14 and 16), we speculate instead that a long-range interaction with Haro 4 is responsible for the warp, with Haro 4 pulling the near side of the disk of Haro 26 toward it. A similar case of a tidal interaction between the BCD SBS 0335-052 and the galaxy NGC 1376 was proposed by Pustilnik et al. (2001a) to explain the burst of star formation in the former. The projected separation between the latter two galaxies is about $150 \mathrm{kpc}$, almost 3 times the projected distance between Haro 4 and
Haro 26. We note that the radio continuum emission of Haro 26 shown in Figure 17 follows the same warp seen in $\mathrm{H}$ I. The intensities of the radio continuum blobs in the south and the northeast warp reach $0.25 \mathrm{mJy}$ beam $^{-1}$.

\subsection{Star Formation}

Star formation is thought to occur whenever the local gas density exceeds a certain threshold. This can be an empirical level, as proposed by Skillman (1987), who found that star formation in dwarf irregular galaxies occurs for densities above $\sim 5 \times 10^{20} \mathrm{~cm}^{-2}$. Alternatively, the local gas density can cause clouds to become gravitationally unstable (Toomre 1964). Taylor et al. (1994) found in their sample that this threshold was equivalent to a column density of $\mathrm{H}$ I of $10^{21} \mathrm{~cm}^{-2}$. Our spatial resolution is insufficient to independently calculate the threshold value for Haro 2 or Haro 4. Qualitatively, star formation activity is seen in our maps above $10^{21} \mathrm{~cm}^{-2}$, and the regions of highest $\mathrm{H}$ I column density coincide with the galaxies seen in the optical. It is worth noting that the $\mathrm{CO}$ in Haro 2 closely follows the highest $\mathrm{H}$ i concentration, indicating that star formation is likely restricted to the very central regions, as reported by Taylor et al. (1994).

The radio continuum also traces the recent star formation activity. In the case of Haro 2 the radio continuum distribution (obtained as a by-product of the $21 \mathrm{~cm}$ spectral line observations) was only marginally resolved and rather featureless. Our $20 \mathrm{~cm}$ radio continuum flux (see Table 5) compares reasonably well with the 20 mJy reported by Beck et al. (2000), who show a much higher resolution map than ours (VLA A configuration) restricted to a zone smaller than the optical galaxy.

Although Haro 2 is metal-poor, its $\mathrm{H}$ II region metallicity at $\sim 0.3 Z_{\odot}$ is not extremely low. This implies that we are not observing its first burst of star formation and supports the suggestion that, whereas the youngest burst began less than 10 Myr ago, it was preceded by other bursts (Fanelli et al. 1988). We can estimate the current rate of formation of massive $\left(M \geq 5 M_{\odot}\right)$ stars using the relations of Condon (1992). In fact, we can use three independent SFR indicators and check if they are mutually consistent.

Based on the $1.4 \mathrm{GHz}$ continuum luminosity and assuming the radio continuum is of nonthermal origin (as argued in $\S 4.1$ ), we obtain from Condon's equation (21)

$$
\frac{\operatorname{SFR}_{1.4}\left(M \geq 5 M_{\odot}\right)}{M_{\odot} \mathrm{yr}^{-1}}=\frac{L_{1.4}}{4.0 \times 10^{21} \mathrm{~W} \mathrm{~Hz}^{-1}},
$$

yielding $\sim 0.28 M_{\odot} \mathrm{yr}^{-1}$ for Haro 2 if we use $S_{1.4 \mathrm{GHz}}$ from Table 5 . Using the $\mathrm{H} \alpha$ luminosity, Condon's equation (22) states

$$
\frac{\operatorname{SFR}_{\mathrm{H} \alpha}\left(M \geq 5 M_{\odot}\right)}{M_{\odot} \mathrm{yr}^{-1}}=\frac{L_{\mathrm{H} \alpha}}{4.4 \times 10^{34} \mathrm{~W}},
$$

yielding $\sim 0.39 M_{\odot} \mathrm{yr}^{-1}$ for Haro 2 , if we use the total $F_{\mathrm{H} \alpha}=3.8 \times 10^{-12} \mathrm{ergs} \mathrm{cm}^{-2} \mathrm{~s}^{-1}$ implied by the narrowband imaging of Méndez \& Esteban (2000). Finally, using the FIR $(40-120 \mu \mathrm{m})$ dust luminosity as obtained by IRAS, Condon's equation (26) gives

$$
\frac{\operatorname{SFR}_{\mathrm{FIR}}\left(M \geq 5 M_{\odot}\right)}{M_{\odot} \mathrm{yr}^{-1}}=\frac{L_{\mathrm{FIR}}}{1.1 \times 10^{10} L_{\odot}},
$$

yielding $\sim 0.33 M_{\odot} \mathrm{yr}^{-1}$ for Haro 2 . Note that we used the IRAS Faint Source Catalog flux densities at 60 and $100 \mu \mathrm{m}$ to derive 
$F_{\text {FIR. }}{ }^{3}$ The good agreement between these three estimates suggests that dust does not severely attenuate Haro 2's observed $\mathrm{H} \alpha$ luminosity (at least along our line of sight). This favorable geometry, together with the disordered $\mathrm{CO}(1-0)$ velocity field in the inner starbursting region, may explain why Haro 2 shows a strong Ly $\alpha$ emission line with a P Cygni profile, as reported by Lequeux et al. (1995) and seen in new HST/STIS data (Mas-Hesse et al. 2003). As we emphasized above, the absence of similar Ly $\alpha$ emission from Haro 4 in the same study may be in part related to that galaxy's much less disturbed velocity field, which renders Ly $\alpha$ photons less able to avoid resonant scattering and escape the galaxy.

\section{SUMMARY}

VLA H I observations of Haro 2 reveal emission extending over a $7.9 \mathrm{kpc} \times 4.0 \mathrm{kpc}$ area, roughly coinciding with the optical galaxy $(5.7 \mathrm{kpc} \times 5.1 \mathrm{kpc})$ in extent and orientation. The $\mathrm{H}$ I mass we see in our interferometric data is $2.3 \times 10^{8} M_{\odot}$; a single-dish spectrum suggests then that the object is embedded in a smooth, extended $\mathrm{H}$ I halo containing an additional $1.3 \times 10^{8} M_{\odot}$ of atomic gas. The $M_{\mathrm{H}_{\mathrm{I}}} / L_{B}$ ratio is about 0.1 in solar units. Surprisingly, the kinematical major axis lies almost perpendicular to the optical (photometric) major axis. This strongly suggest an external source for the $\mathrm{H} \mathrm{I}$ gas. The picture emerging from our study is that of Haro 2 being a dwarf elliptical galaxy (as already postulated by Loose \& Thuan 1986 on the basis of their optical photometry), which has acquired in an interaction or recent merger a substantial amount of gas. Assuming that this gas has settled into regular orbits, we derive a dynamical mass of $2.5 \times 10^{9} M_{\odot}$ and an $M_{\mathrm{dyn}} / L_{B}$ ratio of 1.0. As we have no independent handle on the inclination, the estimate of the dynamical mass is a lower limit and could be larger by a factor of a few. Given the uncertainties involved, this would imply that there is little need to invoke dark matter in Haro 2.

High-resolution, interferometric $\mathrm{CO}(1-0)$ observations with OVRO broadly agree with the $\mathrm{H}$ I data. The molecular gas

\footnotetext{
${ }^{3}$ We would like to mention that the total SFR in Haro 2 must also take into account stars with masses $M<5 M_{\odot}$, the details depending on the choice of initial mass function (IMF). Using the calibration by Kennicutt (1998) for a 0.1-100 $M_{\odot}$ Salpeter (1955) IMF, for example, we estimate $\mathrm{SFR}_{\mathrm{H} \alpha} \simeq 1.37 M_{\odot}$ $\mathrm{yr}^{-1}$ and $\mathrm{SFR}_{\mathrm{IR}} \simeq 0.93 M_{\odot} \mathrm{yr}^{-1}$.
}

coincides with the region of highest $\mathrm{H}$ I column density. The orientation of the $\mathrm{CO}$ emission is aligned with the optical and coincides with published $\mathrm{H} \alpha$ data. The kinematics, although rather disturbed, resembles that of the $\mathrm{H}_{\mathrm{I}}$ velocity field. Assuming a Galactic conversion factor, we detect $7.3 \times 10^{7} M_{\odot}$ of molecular hydrogen.

Haro 4 is unresolved in $\mathrm{H}$ I. We find an $\mathrm{H}_{\mathrm{I}}$ mass of $1.9 \times 10^{7} M_{\odot}$. A $\mathrm{CO}(2-1)$ observation with the $\mathrm{CSO}$ yielded an upper limit to the mass of molecular hydrogen of less than $1.6 \times 10^{7} M_{\odot}$. Haro 4 seems to be interacting with the nearby spiral galaxy Haro 26 (=NGC 3510), the latter object showing a pronounced warp in the direction of the BCD.

Our results provide additional proof that interactions with neighboring galaxies are responsible for the onset of the current episodes of star formation in BCDs. Although the two galaxies under consideration lie in relatively low-density environments, we conclude that a major accretion event or merger can account for the nuclear starburst in Haro 2, as well as for its peculiar kinematics, whereas a more transient encounter between Haro 4 and the neighboring spiral Haro 26 could explain both the star formation in the former and the pronounced warp in the latter.

We thank Lesley Summers for providing the ROSAT HRI image used in Figure 8 and the NRAO for a generous allocation of observing time. We are grateful to the anonymous referee for various thoughtful comments that helped to improve the paper. This research has made use of the NASA/IPAC Extragalactic Database, which is operated for NASA by the Jet Propulsion Laboratory, California Institute of Technology. We have also used the Digital Sky Survey, produced at the Space Telescope Science Institute, and the Lyon-Meudon Extragalactic Database (LEDA) supplied by the LEDA team at the CRALObservatoire de Lyon (France). H. B. A. thanks the CONACyT of Mexico for its support through grant 129987-E; E. B. gratefully acknowledges the award of CONACyT research grant 27606-E. The authors thank the scientific and technical staff at OVRO for making our $\mathrm{CO}$ observations possible; the OVRO millimeter array has been supported in part by National Science Foundation grant AST 93-14079 and the Norris Planetary Origins project. A. J. B. acknowledges support from an NSF Graduate Research Fellowship.

\section{REFERENCES}

Arimoto, N., Sofue, Y., \& Tsujimoto, T. 1996, PASJ, 48, 275

Barone, L. T., Heithausen, A., Hüttemeister, S., Fritz, T., \& Klein, U. 2000, MNRAS, 317, 649

Beck, S. C., Turner, J. L., \& Kovo, O. 2000, AJ, 120, 244

Bender, R., Burstein, D., \& Faber, S. M. 1992, ApJ, 399, 462

Braine, J., Duc, P.-A., Lisenfeld, U., Charmandaris, V., Vallejo, O., Léon, S., \& Brinks, E. 2001, A\&A, 378, 51

Briggs, D. S. 1995, Ph.D. thesis, New Mexico Inst. Mining Tech.

Cairós, L. M., Caon, N., Vílchez, J. M., González-Pérez, J. N., \& MuñozTunón, C. 2001, ApJS, 136, 393

Condon, J. J. 1992, ARA\&A, 30, 575

Doublier, V., Comte, G., Petrosian, A., Surace, C., \& Turatto, M. 1997, A\&AS, 124,405

Fanelli, M. N., O’Connell, R. W., \& Thuan, T. X. 1988, ApJ, 334, 665

Fritz, T. 2000, Ph.D. Thesis, Univ. Bonn

Gerin, M., \& Phillips, T. G. 2000, ApJ, 537, 644

Gondhalekar, P. M., Johansson, L. E. B., Brosch, N., Glass, I. S., \& Brinks, E. 1998, A\&A, 335, 152

Gordon, D., \& Gottesman, S. T. 1981, AJ, 86, 161

Gorjian, V., Turner, J. L., \& Beck, S. C. 2001, ApJ, 554, L29
Haynes, M. P., Giovanelli, R., Chamaraux, P., da Costa, L. N., Freudling, W., Salzer, J. J., \& Wegner, G. 1999, AJ, 117, 139

Hollenbach, D. J., Werner, M. W., \& Salpeter, E. E. 1971, ApJ, 163, 165

Huchtmeier, W. K., Sage, L. J., \& Henkel, C. 1995, A\&A, 300, 675

Hunter, S. D., et al. 1997, ApJ, 481, 205

Israel, F. P., Tacconi, L. J., \& Baas, F. 1995, A\&A, 599, 604

Izotov, Y. I., \& Thuan, T. X. 1999, ApJ, 511, 639

Jörsäter, S., \& van Moorsel, G. A. 1995, AJ, 110, 2037

Kennicutt, R. C., Jr. 1998, ARA\&A, 36, 189

Kobulnicky, H. A., \& Skillman, E. D. 1995, AJ, 454, L121

Kunth, D., \& Joubert, M. 1985, A\&A, 142, 411

Kunth, D., Mas-Hesse, J. M., Terlevich, E., Terlevich, R., Lequeux, J., \& Fall, S. M. 1998, A\&A, 334, 11

Kunth, D., \& Östlin, G. 2000, A\&A Rev., 10, 1

Lee, J. C., Salzer, J. J., Impey, C., Thuan, T. X., \& Gronwall, C. 2002, AJ, 124,3088

Legrand, F., Kunth, D., Mas-Hesse, J. M., \& Lequeux, J. 1997, A\&A, 326,929

Lequeux, J., Kunth, D., Mas-Hesse, J. M., \& Sargent, W. L. W. 1995, A\&A, 301, 18 
Loose, H.-H., \& Thuan, T. X. 1986, ApJ, 309, 59

Mac Low, M.-M., \& Ferrara, A. 1999, ApJ, 513, 142

Martin, C. L., Kobulnicky, H. A., \& Heckman, T. M. 2002, ApJ, 574, 663

Mas-Hesse, J. M., \& Kunth, D. 1999, A\&A, 349, 765

Mas-Hesse, J. M., Kunth, D., Tenorio-Tagle, G., Leitherer, C., Terlevich, R. J., \& Terlevich, E. 2003, ApJ, 598, 858

Meier, D. S., Turner, J. L., \& Beck, S. C. 2001a, AJ, 122, 1770 . 2002, AJ, 124, 877

Meier, D. S., Turner, J. L., Crosthwaite, L. P., \& Beck, S. C. 2001b, AJ, 121,740

Melnick, J., Heydari-Malayeri, M., \& Leisy, P. 1992, A\&A, 253, 16

Méndez, D. I., \& Esteban, C. 2000, A\&A, 359, 493

Möllenhoff, C., Hummel, E., \& Bender, R. 1992, A\&A, 255, 35

Nilson, P. 1973, Uppsala General Catalogue of Galaxies (Uppsala: Uppsala Astron. Obs.)

Noeske, K. G., Iglesias-Páramo, J., Vílchez, J. M., Papaderos, P., \& Fricke, K. J. 2001, A\&A, 371, 806

Oosterloo, T. A., Morganti, R., Sadler, E. M., Vergani, D., \& Caldwell, N. 2002, AJ, 123, 729

Östlin, G. 2000, ApJ, 535, L99

Ott, J., Martin, C. L., \& Walter, F. 2003, ApJ, 594, 776

Padin, S., Scott, S. L., Woody, D. P., Scoville, N. Z., Seling, T. V., Finch, R. P., Giovanine, C. J., \& Lawrence, R. P. 1991, PASP, 103, 461

Papaderos, P., Loose, H.-H., Fricke, K. J., \& Thuan, T. X. 1996, A\&A, 314, 59

Pustilnik, S. A., Brinks, E., Thuan, T. X., Lipovetsky, V. A., \& Izotov, Y. I. 2001a, AJ, 121, 1413

Pustilnik, S. A., Kniazev, A. Y., Lipovetsky, V. A., \& Ugryumov, A. V. 2001b, A\&A, 373, 24

Rosolowsky, E., Plambeck, R., Engargiola, G., \& Blitz, L. 2003, ApJ, 599, 257

Sage, L. J., Salzer, J. J., Loose, H.-H., \& Henkel, C. 1992, A\&A, 265, 19

Salpeter, E. E. 1955, ApJ, 121, 161

Salzer, J. J., Rosenberg, J. L., Weisstein, E. W., Mazzarella, J. M., \& Bothun, G. D. 2002, AJ, 124, 191

Sandage, A., \& Bedke, J. 1994, The Carnegie Atlas of Galaxies, Volume 1 (Washington: Carnegie Inst.)

Sanders, D. B., Scoville, N. Z., \& Soifer, B. T. 1991, ApJ, 370, 158

Scoville, N. Z., Carlstrom, J. E., Chandler, C. J., Phillips, J. A., Scott, S. L., Tilanus, R. P. J., \& Wang, Z. 1993, PASP, 105, 1482
Searle, L., \& Sargent, W. L. W. 1972, ApJ, 173, 25

Shepherd, M. C. 1997, in ASP Conf. Ser. 125, Astronomical Data Analysis Software and Systems VI, ed. G. Hunt \& H. E. Payne (San Francisco: ASP), 77

Skillman, E. D. 1987, in Star Formation in Galaxies, ed. C. J. L. Persson (Washington: GPO), 263

Smoker, J. V., Davies, R. D., Axon, D. J., \& Hummel, E. 2000, A\&A, 361, 19

Strong, A. W., et al. 1988, A\&A, 207, 1

Summers, L. K., Stevens, I. R., \& Strickland, D. K. 2001, MNRAS, 327, 385

Taylor, C. L. 1997, ApJ, 480, 524

Taylor, C. L., Brinks, E., Pogge, R. W., \& Skillman, E. D. 1994, AJ, 107, 971

Taylor, C. L., Hüttemeister, S., Klein, U., \& Greve, A. 1999, A\&A, 349, 424

Taylor, C. L., Kobulnicky, H. A., \& Skillman, E. D. 1998, AJ, 116, 2746

Toomre, A. 1964, ApJ, 139, 1217

Tifft, W. G., \& Cocke, W. J. 1988, ApJS, 67, 1

Tosi, M. 2001, in The Magellanic Clouds and Other Dwarf Galaxies, ed. K. S. De Boer, R.-J. Dettmar, \& U. Klein (Aachen: Shaker), 67

Tremonti, C. A., Calzetti, D., Leitherer, C., \& Heckman, T. M. 2001, ApJ, 555,322

Tully, R. B. 1988, Nearby Galaxies Catalog (Cambridge: Cambridge Univ. Press)

Turner, J. L., Beck, S. C., Crosthwaite, L. P., Larkin, J. P., McLean, I. S., \& Meier, D. S. 2003, Nature, 423, 621

Turner, J. L., Beck, S. C., \& Ho, P. T. P. 2000, ApJ, 532, L109

Turner, J. L., Beck, S. C., \& Hurt, R. L. 1997, ApJ, 474, L11

Turner, J. L., Ho, P. T. P., \& Beck, S. C. 1998, AJ, 116, 1212

van Moorsel, G., Kemball, A., \& Greisen, E. 1996, in ASP Conf. Ser. 101, Astronomical Data Analysis Software and Systems V, ed. G. H. Jacoby \& J. Barnes (San Francisco: ASP), 37

van Zee, L., Salzer, J. J., \& Skillman, E. D. 2001, AJ, 122, 121

van Zee, L., Skillman, E. D., \& Salzer, J. J. 1998, AJ, 116, 1186

Vidal-Madjar, A., et al. 2000, ApJ, 538, L77

Walter, F., Brinks, E., Duric, N., \& Klein, U. 1997, AJ, 113, 2031

Walter, F., et al. 2004, in preparation

Walter, F., Taylor, C. L., Hüttemeister, S., Scoville, N., \& McIntyre, V. 2001, AJ, 121, 727

Walter, F., Weiss, A., Martin, C., \& Scoville, N. 2002, AJ, 123, 225

Wilson, C. D. 1995, ApJ, 448, L97 